\title{
Fremdsprachenausbildung - Bedarfs- und Erfahrungsanalyse der Fremdsprachenausbildung in der slowenischen öffentlichen Verwaltung
}

UDK: 37:81'243:35(497.4)

Margit Horvath

Universität Ljubljana, Fakultät für Verwaltungswissenschaften margith.horvath@fu.uni-li.si

Manica Danko

Universität Ljubljana, Fakultät für Verwaltungswissenschaften manica.danko@fu.uni-li.si

Polonca Kovač

Universität Ljubljana, Fakultät für Verwaltungswissenschaften polona.kovač@fu.uni-li.si

Janez Stare

Universität Ljubljana, Fakultät für Verwaltungswissenschaften janez.stare@fu.uni-li.si

AUSZUG

Während der Bedarf an Fremdsprachenausbildung und Selbststudium in der Privatwirtschaft fast ein Muss ist, ist die Situation in der öffentlichen Verwaltung verhältnismäßig unbekannt. Mit Hilfe der Forschung verschiedener Meinungen und Erfahrungen, wollten wir den Bedarf der Verwaltungsangestellten an Fremdsprachenkenntnissen und Sprachenkompetenzen (Schreiben, Sprechen, Hören und Lesen) am Arbeitsplatz überprüfen. Die Bedarfs- und Erfahrungsanalyse der slowenischen öffentlichen Verwaltung im Bereich Horvat, M., Danko, M., Kovač, P. \& Stare, J. (2012). Fremdsprachenausbildung Bedarfs- und Erfahrungsanalyse der Fremdsprachenausbildung in der slowenischen öffentlichen Verwaltung. Uprava X(1), 105-143. 
der Fremdsprachenausbildung zeigte, dass Verwaltungsangestellte Kenntnisse der englischen (83\%) und deutschen Sprache (68\%) benötigen, während der Bedarf an Kenntnissen der französischen Sprache trotz der engeren internationalen Zusammenarbeit der slowenischen öffentlichen Dienste mit den EU-Institutionen um gut ein Viertel $(26,22 \%)$ niedriger ist als an der kroatischen und serbischen Sprache, die wegen der Einwanderung der Staatsbürger aus dem Gebiet des ehemaligen Jugoslawien in gut der Hälfte der befragten Organisationen benötigt werden. Der Bedarf an Fremdsprachenkenntnissen ist an verschiedenen Arbeitsplätzen vorhanden: die Fremdsprachenkenntnisse benötigen sowohl leitende Verwaltungsangestellte als auch Berater, (Staats-)Sekretären, Beamten, die für Ausländer und internationale Zusammenarbeit verantwortlich sind. Der größte Bedarf am Fremdsprachenerwerb steht im Zusammenhang mit der Zusammenarbeit Sloweniens mit der EU (Angestellte der öffentlichen Verwaltung benötigen Fremdsprachen am häufigsten für die Teilnahme an den EU-Ausschreibungen), der Verfolgung der europäischen Gesetzgebung und der internationalen Zusammenarbeit (z.B. bei der Leitung von internationalen Arbeitsgruppen oder bei der Kommunikation mit benachbarten Gemeinden). Der Bedarf an Fremdsprachenkenntnissen ist geringer im Bereich der Rechtspflege, Steuern, Sachgebiet- und Protokollentwicklung und der Kommunikation mit den Kunden aus dem Ausland.

\section{Schlüsselbegriffe: Fremdsprachen, Fremdsprachenausbildung, Verwaltungsangestellte, Fremdsprachenbedarf}

JEL: $100,1121,200$

\section{Einleitung}

Die Kenntnisse der slowenischen und anderen Fremdsprachen sowie deren Bedarfsermittlung in der öffentlichen Verwaltung, werden wegen der Umwandlung der slowenischen öffentlichen Verwaltung und der Integration in das europäische Verwaltungssystem, immer wichtiger. Der Artikel 61 der Verfassung der Republik Slowenien legt fest, dass »jedem das Recht zusteht, seine National- und Volkszugehörigkeit frei zu bekennen, seine Kultur zu pflegen und seine Sprache und Schrift zu gebrauchen «'. Der Fremdsprachengebrauch ist bei der Ausübung amtlicher Tätigkeiten dann erlaubt, wenn im Verfahren vor den Staatsbehörden und

1 Einen solchen rechtlichen Rahmen bestätigte auch das Gesetz über den Gebrauch der slowenischen Sprache, das in der öffentlichen Verwaltung außer dem Gebrauch des Slowenischen als Amtssprache, auch den Gebrauch des Ungarischen und Italienischen auf den Gebieten der Minderheiten (Artikel 3) sowie anderer Fremdsprachen bei amtlichen Tätigkeiten (Artikel 5), internationaler Zusammenarbeit (Artikel 6) und Kundenkontakten auf geschäftlicher Ebene (Artikel 14) vorsieht. 
Margit Horvath, Manica Danko, Polonca Kovač, Janez Stare

Fremdsprachenausbildung - Bedarfs- und Erfahrungsanalyse der Fremdsprachenausbildung in der slowenischen öffentlichen Verwaltung

Behörden der lokalen Selbstverwaltung, Ausführenden der öffentlichen Dienste und Trägern der öffentlichen Befugnisse, ausländische juristische Personen sind. In diesem Fall kann außer dem Slowenischen auch eine Fremdsprache rechtmäßig verwendet werden. Die ungarische und italienische Sprache haben wegen der Verfassungskautelen der geschützten nationalen Minderheiten Sonderschutz (mehr in Šturm et al., 2011 ), obwohl in Slowenien die Zahl der Einwohner, die Ungarisch und Italienisch sprechen, relativ gering ist. Das geht auch aus der vorliegenden Analyse hervor, da die Fremdsprachenausbildung auf den Gemeindegebieten, wo die beiden Sprachen Amtssprachen sind (siehe Jerovšek \& Kovač, S. 101-104), sich vom Bedarf anderswo nicht wesentlich unterscheidet. Die Fremdsprache wird auch bei der internationalen Zusammenarbeit öffentlicher Dienste sowie bei einigen anderen ähnlichen Formen verwendet, wenn das im Regionalgesetz besonders festliegt. Bei Kundenkontakten zwischen Rechtspersonen auf geschäftlicher Ebene in der Republik Slowenien, wird der Gebrauch der Fremdsprache erlaubt, wenn Angestellte im öffentlichen Dienst mit den Kunden aus dem Ausland in Kontakt kommen bzw. ihre Arbeit mit Ausländern zusammenhängt.

Die Unterstützung der Sprachenvielfalt ist einer der Grundsätze der EUArbeitsweise. Die Anzahl der Amtssprachen der EU stieg durch die Erweiterungen 2004 und 2007 von 11 auf 23². In Übereinstimmung mit der Sprachenpolitik fordert die EU, dass ihre Gesetzgebung in allen Amtssprachen zur Verfügung steht und somit allen Bürgern zugänglich ist. Das bedeutet, dass sich Vertreter der erwähnten Amtssprachen bei offiziellen Tagungen in den EU-Institutionen in ihrer Sprache verständigen können und in ihre Sprache auch alle Rechtsakte und das Amtsblatt übersetzt werden sollten. Trotzdem wird wegen Zeitnot und zu hoher Kosten nur eine geringe Zahl der offiziellen Dokumente in alle Amtssprachen übersetzt. Diese werden hauptsächlich in jene Sprachen übersetzt, in denen die Kommunikation meistens abläuft, z.B. in der Europäischen Kommission in die englische, französische und deutsche Sprache. Das Europäische Parlament gewährleistet Übersetzungen in die drei o.g. Sprachen, während die Dokumente in andere Amtssprachen nur nach Bedarf der Abgeordneten übersetzt werden.

2 Englisch, Bulgarisch, Tschechisch, Dänisch, Estnisch, Finnisch, Französisch, Griechisch, Irisch, Italienisch, Lettisch, Litauisch, Ungarisch, Maltesisch, Deutsch, Holländisch, Polnisch, Portugiesisch, Rumänisch, Slowakisch, Slowenisch, Spanisch und Schwedisch. 
Mit der EU-Mitgliedschaft übernahm Slowenien die Grundsätze, dass Bürger aus den EU-Mitgliedsländern keine Arbeits- oder Aufenthaltserlaubnis brauchen (Verlič Christensen, 2000, S. 1127). Das bedeutet, dass wir als gleichberechtigtes Mitglied das Prinzip der Menschenrechte im Zusammenhang mit der Freizügigkeit innerhalb des Gebietes der EU-Länder beachten müssen. Darüber hinaus kann sich jeder EU-Bürger in jedem beliebigen Mitgliedsland in seiner eigenen Sprache bzw. irgendeiner anderen Amtssprache der EU verständigen (EC.Europa, 2009).

Nach den Angaben des Statistischen Amtes der Republik Slowenien aus dem Bericht über die Umzugsbewegungen nahm seit dem Jahr 2000 die Zahl der zu uns eingewanderten Ausländer jährlich ständig zu, besonders nach dem Jahr 2004 als sich die Zahl jährlich in der Regel um 50\% erhöhte. Nach dem Jahr 2008 nahm die Zahl im Vergleich zu den vergangenen Jahren allmählich ab. Im Jahr 2009 wanderten nach Slowenien 30.296 Einwohner ein (das ist etwas weniger als 2008), davon 27.393 ausländische und 2.903 slowenische Staatsbürger. Der Grund dafür lag in der Krise im Baugewerbe, in dem in den vorigen Jahren die höchste Zahl der zu uns eingewanderten ausländischen Staatsbürger angestellt war.

Unter den ausländischen Staatsbürgern, die zu uns im Jahr 2009 eingewandert sind, waren vor allem Staatsbürger aus Bosnien und Herzegowina (und zwar 12.900 bzw. 47\% von allen eingewanderten Ausländern). Die zweitgrößte Gruppe darunter waren die Staatsbürger aus Kosovo (fast 3.600 bzw. 13,1\% aller eingewanderten Ausländer). Die Staatsbürger dieses neven Staates, der im Jahr 2008 gegründet worden ist, wurden in den Angaben aus den vergangenen Jahren als serbische Staatsbürger berücksichtigt. In Folge der Unabhängigkeit des Kosovo im Jahr 2009 sank entsprechend die Zahl der eingewanderten Staatsbürger aus Serbien, die noch im Jahr 2008 die zweitgrößte Gruppe der eingewanderten Ausländer waren. Im Jahr 2009 wanderten so nach Slowenien etwas mehr als 2.900 Staatsbürger aus Serbien ein ${ }^{3}$. Unter den eingewanderten Ausländern gab es im Jahr 2009 1.881, das ist um 9\%

3 Unter den Ausländern, die nach Slowenien eingewandert sind, gab es im Jahr 2007 85,4\% der Staatsbürger aus Ländern aus dem Gebiet des ehemaligen Jugoslawien: vor allem aus Bosnien und Herzegowina (12.479), Serbien (6.368) und Mazedonien (3.163). 
Margit Horvath, Manica Danko, Polonca Kovač, Janez Stare

Fremdsprachenausbildung - Bedarfs- und Erfahrungsanalyse der Fremdsprachenausbildung in der slowenischen öffentlichen Verwaltung

weniger als im Jahr 2008. Darunter war die Mehrzahl aus Bulgarien (539), Italien (271) und der Slowakei (208) (Statistisches Amt, 2011).

Der Bedarf an der Fremdsprachenausbildung in Slowenien kam in der Vergangenheit am häufigsten aufgrund einiger allgemeiner Migrationseigenschaften Sloweniens zum Ausdruck. Slowenien galt schon 30 Jahren vor seiner Unabhängigkeit als wichtiges Einwanderungsgebiet des ehemaligen Jugoslawien. Diese Rolle bewahrte es auch nach dem Umbruchiahr 1991. Seit der Unabhängigkeit gehören zu den zahlreichsten Einwanderern ausländische Staatsbürger aus dem Gebiet des ehemaligen Jugoslawien, besonders aus Bosnien und Herzegowina. Denen folgen in unterschiedlicher Reihenfolge und vom Jahrgang abhängig die Staatsbürger aus dem ehemaligen Serbien und Montenegro, Kroatien und Mazedonien. Zwischen den Jahren 1996 und 2006 ging der Anteil der Staatsangehörigen aus dem ehemaligen Jugoslawien um 5,5 Prozent auf Kosten von den EU-Bürgern und Nachfolgern der Sowjetunion zurück. Im Mai 2006 nahm Slowenien die Arbeitnehmerfreizügigkeit für die Bürger aus allen Ländern der EU und des Europäischen Wirtschaftsraums in Anspruch. Der größte Teil der Einwanderer kam zu uns, um eine Beschäftigung oder Saisonarbeit zu finden, darunter gab es am meisten Männer. Slowenien wurde Anfang der 90-er Jahre, ähnlich wie andere Länder am westlichen Rand der damaligen EU, zU einem wichtigen Durchgangsstaat. Dabei spielte seine bereits traditionelle Verbindungslage zwischen den Balkanstaaten und dem Mittel- bzw. Westeuropa die entscheidende Rolle (Komac, 2007, Seiten 464-467).

Der Beitrag behandelt die Bedarfs- und Erfahrungsanalyse der Fremdsprachenausbildung (der Angestellten in der öffentlichen Verwaltung) mit der Absicht dieses Gebiet zu erforschen und die Art und Stärke des Einzelbedarfs zu definieren.

\section{2 Öffentliche Verwaltung und Fremdsprachenpraxis}

Um sich ein besseres Verständnis für den Sprachbedarf zu verschaffen, können verschiedene Aktivitäten bzw. Gründe für den Fremdsprachenbedarf bestimmt werden. Am häufigsten kommen sie beispielsweise durch touristische, geschäftliche, forschungsbezogene (Immobilieneigentum, Sorgerecht, Pflegschaft, ...) oder arbeitsbezogene Tätigkeiten (grenzüberschreitende Dienstleistungserbringung mit eingewiesenen 
Arbeitern, Saisonarbeit, ...) zum Ausdruck. Sie davern unterschiedlich lang, was auch die Aktivitäten der Ausländer bzw. ihre Kontakte zu unseren Behörden wesentlich beeinflusst. Während seines Aufenthalts in Slowenien kann ein Ausländer z.B. bei der Verwaltungsbehörde Verwaltungsangelegenheiten wie Einreise nach Slowenien, Aufenthalt in der Republik Slowenien, Integration, Erledigung der Staatsangehörigkeit und Flüchtlinge und Personen mit vorübergehendem Schutz erledigen. Einer der häufigen Gründe für den Bedarf an Fremdsprachenkenntnissen in der öffentlichen Verwaltung sind Einwanderer. Der Großteil der Einwanderer wanderte wegen einer Anstellung oder Saisonarbeit ein (Komac, 2007, S. 466).

Bei der Kommunikation der Amtspersonen mit den ausländischen Kunden, die weder Slowenisch noch Italienisch oder Ungarisch können, kann es bei der Ausführung und Lösung von Verwaltungsangelegenheiten zu Verständigungsschwierigkeiten kommen, wenn es keinen Angestellten gibt, der die entsprechende Fremdsprache spricht. In solchen Fällen gibt es mehrere Lösungen, die bei der Aufklärung von Missverständnissen wegen der Fremdsprachenhindernisse behilflich sind. Einen ausländischen (fremdsprachigen) Kunden kann eine Person begleiten, die seine Sprache entweder in die slowenische oder jene Sprache übersetzen wird, die die Amtsperson aktiv oder passiv versteht.

Darüber hinaus ist es nicht unbedingt notwendig, dass alle Probleme, die mit den Fremdsprachenkenntnissen zusammenhängen, auf amtliche Angelegenheiten der Angestellten in der öffentlichen Verwaltung in Hinsicht auf Kunden aus dem Ausland zurückzuführen sind. $\mathrm{Zu}$ Schwierigkeiten kann es auch kommen, wenn die Verwaltungsangestellten an internationalen Projekten oder EU-Ausschreibungen teilnehmen oder einfach täglich amtliche EU-Dokumente lesen, die in den meisten Fällen in die englische, französische oder deutsche (und sehr selten slowenische) Sprache übersetzł sind. 
Margit Horvath, Manica Danko, Polonca Kovač, Janez Stare

Fremdsprachenausbildung - Bedarfs- und Erfahrungsanalyse der Fremdsprachenausbildung in der slowenischen öffentlichen Verwaltung

\section{1 Über Kompetenzen der Verwaltungsangestellten im Bereich der internationalen Tätigkeiten}

Das Kompetenzmodell für das Management (die Führung) in der Staatsverwaltung ${ }^{4}$ besteht aus sieben Schlüsselkompetenzen ${ }^{5}$, die sich auf drei Ebenen auf leitende Angestellte im öffentlichen Dienst beziehen: Generalsekretäre, Generaldirektoren der Direktionen, Leiter der den Ministerien unterstellten Behörden, Leiter der Regierungsdienste, Leiter der Verwaltungsbehörden (erste Ebene), Sektionsleiter, Leiter der Dienststellen (zweite Ebene), Abteilungs- und Referatsleiter (dritte Ebene). Die Untersuchung ergab (Stare, 2007), dass das Kompetenzprofil der Führung auf allen drei Ebenen Bewertungsmaßstäbe anlegt, die bei allen sieben Schlüsselkompetenzen im Vergleich zu den Führungsbewertungen einiger anderer Modelle verhältnismäßig hoch sind. Unter den erwähnten Kompetenzen stellt die Kommunikation in slowenischer Sprache eine der Dimensionen der "Arbeitsflexibilität" dar. Es geht um die Fähigkeit, die gleiche Sprache wie Kunden zu sprechen und jene Kommunikationswege zu wählen, um die Kundenzustimmung zu erreichen und für sie eine qualitativ hochwertige Dienstleistung zu erbringen.

Wegen der Globalisierung des Geschäftsverkehrs, der grenzüberschreitenden Zusammenarbeit, der Geschwindigkeit des Informationszugangs und der Informationsübertragung spielt die internationale Zusammenarbeit heutzutage eine immer wichtigere Rolle. Die Organisationen in der öffentlichen Verwaltung sind auf diesem Gebiet neven Herausforderungen gegenübergestellt, für deren Beherrschung die Verwaltungsangestellten andere Kenntnisse und Fertigkeiten von denjenigen brauchen, die ihnen in der einheimischen Umgebung erfolgreiche und hochwertige Tätigkeiten gewährleisten. Darunter sind die Fremdsprachenkenntnisse eine der wichtigen Kompetenzen. Das sind die Soft Skills ${ }^{6}$, die in der heutigen unvorhersehbaren Zeit Erfolg bei

\footnotetext{
4 Vom Gesichtspunkt der öffentlichen Verwaltung stellt die Staatsverwaltung jenes Segment der slowenischen öffentlichen Verwaltung dar, das die Förderung und Erzielung ihrer Effizienz ausschlaggebend beeinflusst.

5 Arbeitsflexibilität, Kreativität, Management, Organisationsklima, organisatorische Durchführung, Vernetzung und Beeinflussung, Realisierungsfähigkeiten

6 Soft Skills sind persönliche, versteckte, formlose Kenntnisse, verinnerliche Fähigkeiten, die man durch Erfahrungen und Übung erwirbt. Es geht um die Kommunikations- und Verhandlungsfertigkeiten, Vernetzungs- und Beeinflussungsfähigkeit, Sprachkenntnis der interkulturellen Kommunikation, Verwendung und Geschwindigkeit der Kenntnisanwendung (Svetličič, 201 1a, S. 125-126).
} 
der Zusammenarbeit (besonders kleiner Staaten) auf dem internationalen Markt ermöglichen. Während die Hard Skills (formelle Ausbildung) dem breiteren Kreis immer zugänglicher werden, machen die Soft Skills den Unterschied zwischen den Leuten, die nur Wissen haben und denjenigen, die ihre Kenntnisse verwenden und durchsetzen und dazu fähig sind, sie auch erfolgreich zum Ausdruck zu bringen (Svetličič, 2011 a, S. 123-130). Besonders wichtig sind die Verhandlungsfähigkeiten, weil zwischen den Firmen und Regierungen Machtverhältnisse, Kompromisse, Zusammenarbeit und Wettbewerbsfähigkeit herrschen, die die Verhandlungen beeinflussen (Svetličič, 2011b, S. 61). Die Analyse der slowenischen Ratspräsidentschaft ergab deutlichen Mangel an den Soft Skills, wie Englischkenntnisse, Rhetorik, Kontaktaufnahme und Gruppenarbeit (Svetličič \& Krajnc, 2009 in Svetličič, 2011 a, S. 130).

Die Verwaltungsangestellten, die bei der internationalen Zusammenarbeit tätig sind, sind wie Wirtschaftsdiplomaten, die bei der bilateralen, regionalen und multilateralen Durchsetzung von Wirtschaftsinteressen eine immer wichtigere Rolle spielen, um nationale Interessen zu maximieren bzw. den Bevölkerungswohlstand zu steigern. Um entsprechende Informationen zu bekommen, sind außer interdisziplinären Fachkenntnissen auch Kommunikationsfähigkeiten in der Fremdsprache nötig. Svetličič, (201 1a, S. 124) stellt fest, dass die erfolgreiche Kommunikation in der Fremdsprache ein unmittelbarer Wettbewerbsvorteil ist, besonders im Kontakt mit Kulturen, die einen großen Wert auf ihre Identität legen. Schon die Grundkenntnisse in der Sprache der Einheimischen können die Tür zur Geschäftstätigkeit öffnen, oder wenigstens ihre Entwicklung auslösen bzw. beschleunigen und dadurch zur Stärkung der Wettbewerbsvorteile beitragen. Die Beherrschung interkultureller Unterschiede wird zu einer der wichtigsten Internationalisierungskompetenzen (Svetličič, 2011 b, S. 618).

\subsection{Ist-Zustandsaufnahme der Fremdsprachenausbildung in Slowenien}

In den letzten Jahren wurden in Slowenien im Bereich des Fachfremdsprachenunterrichts im Rahmen der Hochschulprogramme mehrere Untersuchungen durchgeführt, die sich mit den folgenden Themen befassten: deutsche Sprache als Lehrfach an der Universität Maribor, Universität Ljubljana und Universität Primorska (Grmek \& Rejec, 2005), Rolle der englischen Fachsprache in den umgestalteten 
Margit Horvath, Manica Danko, Polonca Kovač, Janez Stare

Fremdsprachenausbildung - Bedarfs- und Erfahrungsanalyse der Fremdsprachenausbildung in der slowenischen öffentlichen Verwaltung

Bologna-Studiengängen (Jakoš, 2007), Lage des Fachfremdsprachenunterrichts und der Fachfremdsprachlektoren im slowenischen Hochschulwesen (Jurković, Djurić \& Godnič-Vičič 2007 und 2008), Status der Fach-fremdsprachen innerhalb der zur Anpassung an die BolognaRichtlinien umstrukturierten Programme in Slowenien (Svetlin-Gvardjančič, 2008) und studienbegleitender Deutschunterricht im Rahmen der Bologna-Reform in Slowenien (Horvath, 2008). Ende des vorigen Jahrhunderts beschäftigten sich noch zwei Studien mit der Lage der englischen Sprache an den Mitgliedsinstitutionen der Universität in Ljubliana (Čebron, 1998) und dem Inhalt der Programme für den Fachfremdsprachenunterricht innerhalb verschiedener Diplomstudienrichtungen in Slowenien (Djurić, 1999). Im Jahr 2008 wurde innerhalb des Slowenischen Fachfremdsprachenlehrervereins (SDUTSJ7) die Gruppe zur Bildung der Fremdsprachenpolitik in Slowenien gegründet, die einen Bericht über den Fremdsprachenunterricht auf allen Niveaus in Slowenien vorbereitete $^{8}$.

\subsubsection{Fremdsprachenunterricht an Grund- und Mittelschulen}

Das slowenische Erziehungs- und Ausbildungssystem sieht die erste Fremdsprache erst in der 4. Klasse der Grundschule vor. Deshalb bieten viele Grundschulen im ersten Erziehungs- und Ausbildungsabschnitt Fremdsprachenkurse in eigener Regie an, die von den Eltern entweder ganz oder wenigstens zum Teil finanziert werden müssen. Das bedeutet, dass einige Kinder abgesehen von ihren Wünschen, Fähigkeiten und Ambitionen, wegen ihrem sozialen Status diskriminiert sind. Deswegen entsteht immer wieder das bekannte Problem der s.g. "ewigen Anfänger", die bis Ende der Grundschulausbildung bis zu viermal dieselbe Fremdsprachenstufe lernen, ungeachtet davon wie geschickt sie darin schon sind. Der Fremdsprachenunterricht in der Grundschule ist nicht genug auf die Sprachanwendung ausgerichtet und das Konzept der externen Prüfung ist zu sehr auf die statistischen Sprachkenntnisse und nicht auf ihre Anwendung konzentriert. Zu große Gruppen ermöglichen keine innere Binnendifferenzierung.

7 Slovensko društvo učiteljev tujega strokovnega jezika

8 Der Artikel der Gruppe für die Fremdsprachenpolitik beim SDUTSJ unter dem Titel "Überblick der Folgen von der Abwesenheit der Fremdsprachenpolitik auf allen Ausbildungsniveaus in der Republik Slowenien und Verbesserungsvorschläge des Ist-Zustandes", zugänglich auf http://www.sdutsj.edus.si/tujejezikovna_politika.html, (16. 1. 2012). 
Bei dem Fremdsprachenunterricht im Sekundärschulbereich kommen oft ähnliche Schwierigkeiten wie im Grundschulbereich zum Ausdruck. Einige Mittelschüler fangen wieder an, die zweite Fremdsprache mit den Anfängern zu lernen, weil einige Mittelschulen keine Gruppen für Fortgeschrittene haben. Seitdem die Reform der Berufs- und fachbezogenen Ausbildung in Kraft getreten ist, hat die Mehrheit der drei- und vieriährigen Mittelschulprogrammen nur noch eine Fremdsprache als Pflichtfach, während nur wenige Mittelschüler die Möglichkeit haben, eine zweite Fremdsprache im beschränkten Umfang zu wählen. Die zweite Fremdsprache ist nur noch in einigen fachbezogenen Programmen notwendig. Sogar an Gymnasien mit ausreichender Schülerzahl ist die Möglichkeit der Fremdsprachwahl aus Organisations-, Finanz- und Personalgründen begrenzt.

\subsubsection{Fremdsprachenunterricht an Universitäten}

Auch beim Fremdsprachenunterricht an Universitäten treten verschiedene Problemen auf. Die Fremdsprachen werden in den Studiengängen im unterschiedlichen Umfang von Leistungspunkten (zwischen 3 und 14) und mit sehr unterschiedlichem Verhältnis zwischen Lehrstunden und individueller Arbeit angeboten. Auffallend ist der Mangel an Richtlinien für die Inhaltserstellung, was zur Folge hat, dass die Lernplanerstellung beliebig ist. In den meisten Lernplänen werden das Ausgangs- und Abschlussniveau der Fremdsprachenkenntnisse gemäß dem Gemeinsamen europäischen Referenzrahmen für Sprachen (GeR) nicht definiert. Außerdem kommt es vor, dass Fremdsprachen in die Studiengänge beliebig eingeschlossen werden, d.h. aufgrund der Leistungspunkte, die in den einzelnen Semestern/Jahrgängen verfügbar oder übriggeblieben sind. Ein weiteres Problem stellt der Verlust der Fachfremdsprache in einigen Bologna-Programmen im Vergleich zu den "alten" Hochschulprogrammen dar, sowie die Tatsache, dass es recht viele Studiengänge der BachelorEbene ohne Fremdsprachen gibt.

An den meisten Fakultäten kann man zwischen zwei Fremdsprachen (Englisch und Deutsch) wählen und an einigen wird nur Englisch angeboten. Selten findet man Hochschuleinrichtungen mit drei Fremdsprachen. In der Regel wird die Fremdsprache als Pflichtfach angeboten, und zwar in den meisten Bologna-Studiengängen nur am Anfang des Studiums (normalerweise in dem 1. Jahrgang), oder noch schlimmer erst im dritten oder vierten Jahrgang der Bachelor-Ebene bzw. 
Margit Horvath, Manica Danko, Polonca Kovač, Janez Stare

Fremdsprachenausbildung - Bedarfs- und Erfahrungsanalyse der Fremdsprachenausbildung in der slowenischen öffentlichen Verwaltung

im 2. Jahrgang der Magister-Ebene, wodurch die Kontinuität der Fremdsprachenausbildung unterbrochen wird. Aufgrund der Bologna-Richtlinien kam es nicht zur Erhöhung der Kontaktstundenzahl. Im Rahmen einiger umgesetzter Studiengänge blieb die Zahl der Lehrstunden unverändert, während sie bei den meisten Studiengängen um 25\% abnahm, in einigen Fällen sogar um 65\%. Die Fremdsprache ist stellenweise auch ein Wahlfach, das manchmal bei nicht ausreichender Teilnehmerzahl nicht durgeführt wird. Die Mindestteilnehmerzahl steht nicht genau fest. So sieht das Angebot (Hochschulfremdsprachenunterricht) groß aus, obwohl seine Durchführung öfters fraglich ist. Obwohl in bestimmten Regionen Englisch weniger in Betracht kommt als andere Fremdsprachen, ist das Bewusstseinsniveau über die Vorteile einer zweiten Fremdsprache zu niedrig.

Damit die Bologna-Richtlinien berücksichtigt werden können, ist die Änderung des Inhaltes der Fachfremdsprachenausbildung dringend erforderlich. Obwohl einige Änderungen negativ sind, wie z.B. Weglassung von thematischen Zusammenhängen aufgrund einer geringeren Zahl von Kontaktstunden, kann das auch eine Gelegenheit zur Qualitätssteigerung des Fremdsprachenunterrichts sein (z.B. Lerninhaltsauswahl in der Zusammenarbeit mit den Dozenten anderer Studienfächer, Vorbereitung der Aufgaben im Rahmen von e-Schulungen, um autonomes Lernen auf dem Gebiet des Hör-, Leseverstehens und Schreibens zu fördern, Einleitung der Fallbeispiele und des interkulturellen Lernens).

An Universitäten sind das Ausgangs- und Abschlussniveau der Fremdsprachenkenntnisse nicht definiert, so dass die Gruppen oft heterogen sind und die Prüfungskriterien von den einzelnen Lehrern abhängen. Nach den Einschulungsdaten sind die Gruppen im Durchschnitt aus 30 bis 50 Studierenden zusammengesetzt. Den Studenten verschiedener Studienrichtungen/-gängen wird der Fremdsprachenunterricht teilweise in einer Gruppe angeboten. Wegen der Finanzierung der Bologna-Studiengänge, die sich als insuffizient und nicht entsprechend erwies, sind die Fakultäten dazu gezwungen, die unmittelbare Belastbarkeit der Dozenten (nicht nur für Fremdsprachen) zu verringern, meistens auf Kosten der Erhöhung der Studentenzahl und/oder Gruppenfusion für die Studierenden aus verschiedenen Studiengängen. In den Lehrplänen ist die Gruppengröße nicht fest definiert. Als Folge davon lässt die Arbeitsqualität bei großen Gruppen nach. Zudem wird die Stundenzahl des Präsenzunterrichts reduziert oder sogar die Fachfremdsprache als Studienfach weggelassen. 
Stellenweise sitzen ordentliche und außerordentliche Studierende in den gleichen Gruppen zusammen. Es zeichnet sich auch die Tendenz ab, dass es von Studierenden erwartet wird, dass sie selbst für entsprechendes Niveau ihrer Fremdsprachenkenntnisse im Rahmen der Sprachenkurse sorgen, die in privaten oder öffentlichen Institutionen außerhalb des Studienganges stattfinden und sie selbst bezahlen müssen.

Die Forschungen ergaben zahlreiche Nachteile auf dem Gebiet des Fremdsprachenunterrichts im slowenischen Schulraum, die sich als Folge der Abwesenheit einer nationalen Fremdsprachenpolitik erwiesen. Wenn wir möchten, dass das Lernen von Fremdsprachen von Nutzen ist, müsste das Schulsystemkonzept die Kontinuität des Fremdsprachenunterrichts und die Vernetzung unter den Lehrern auf allen Niveaus der Fremdsprachenausbildung gewährleisten. Die Mittelschulprogramme an Gymnasien und Berufsschulen müssten allgemeine Englischkenntnisse und wenigstens noch eine zweite Fremdsprache beinhalten. Auf Universitätsebene sollte der Fremdsprachenunterricht unbedingt auf die Spezifika der Fachsprachen ausgerichtet sein und in kleineren Gruppen stattfinden. Auf jeder Ausbildungsstufe sollten das Ausgangs- und Zielniveau gemäß dem GeR vorab definiert werden.

\subsection{Bedeutung der Mehrsprachigkeit}

Die Mehrsprachigkeit stellt im europäischen Raum die Voraussetzung für das Zusammenleben von verschiedenen Nationen dar und trägt wesentlich zum Bewusstsein über die Bedeutung der eigenen Sprache in diesem Raum bei. Sie fördert den Gebrauch mehrerer Sprachen beim einzelnen Sprecher wie auch bei der breiteren Gemeinschaft und wird von der Globalisierung bestimmt. Wegen dem einfacheren Zugang zu Internetinformationen, dem Ausgesetztsein von Einzelpersonen verschiedenen Sprachen und Kulturen, dem freien Arbeits-, Waren-, Ausbildungsverkehr und Aufenthalt ist der Bedarf an verschiedenen Sprachkenntnissen (Mehrsprachigkeit) noch größer.

Deshalb waren sich im Jahr 1998 die Bildungsminister der EU darüber einig, dass die Mitgliedstaaten die Mehrsprachigkeit und das frühzeitige Erlernen von Fremdsprachen unterstützen sollten (BMUKK, 2010, 3). Auf die Bedeutung der Mehrsprachigkeit weist auch der GeR hin, der die Notwendigkeit der grundsätzlichen Zielsetzung des heutigen Fremdsprachunterrichts betont. Dieser sollte ein sprachliches Repertoire festsetzen, in dem alle sprachlichen Fähigkeiten gleich wichtig sind. Das Sprachangebot 
Margit Horvath, Manica Danko, Polonca Kovač, Janez Stare

Fremdsprachenausbildung - Bedarfs- und Erfahrungsanalyse der Fremdsprachenausbildung in der slowenischen öffentlichen Verwaltung

der Bildungseinrichtungen muss unterschiedlich sein und den Lernenden eine mehrsprachige Kompetenz ermöglichen. So würden sich die Sprachkenntnisse eines Menschen und seine Verständigung in Fremdsprachen erweitern und verbessern. Außerdem würde die Mehrsprachigkeit die dominante Stellung des Englischen in der internationalen Kommunikation vermindern $(G l, 2001,103)$.

Im Jahr 2000 setzte sich die EU das Ziel, sich bis zum Jahr 2010 zu einer weltweit wettbewerbsfähigen, dynamischen und auf Kenntnissen basierenden Wirtschaft zu entwickeln. Das Ziel ist (war), größere Sozialkohäsion, eine nachhaltige Wirtschaftssteigerung und mehr Arbeitsplätze zu erreichen. Der Plan zur Erreichung dieses Zieles heißt die "LissabonStrategie", in dessen Mittelpunkt die Ausbildungspolitik und das (Fremd)Sprachenlernen stehen. Verschiedene Strategien, zu denen auch das Dokument des EU-Rates »Education and Training 2020« (ET 2020) gehört, legen fest, dass das Lernen von mindestens zweier Fremdsprachen zu den grundlegendsten Aktivitäten einer lernenden Gesellschaft gehören.

In den Studiengängen der Fakultät für Verwaltungswissenschaften werden die Globalisierung und Interkulturalität sehr betont. Daher sind von großer Bedeutung beide Fremdsprachen (Englisch und Deutsch). Die Fremdsprachenlehrer arbeiten eng mit dem internationalen Büro zusammen und wirken auch bei der Organisation für den Austausch von Studierenden und Pädagogen mit, die das Bewusstsein über die Interkulturalität fördern. Im Studienjahr 2011/2012 kontaktierte die Fakultät das Französische Kulturzentrum und veranstaltete mit seiner Hilfe einen Fortgeschrittenenkurs der französischen Sprache, um den internationalen Studienaustausch bzw. die Zusammenarbeit mit den Hochschuleinrichtungen im französischsprachigen Raum zu fördern.

\subsection{Ist-Zustandsaufnahme der Fremdsprachenkenntnisse}

Im Rahmen des slowenischen Ausbildungssystems wird allgemeines Englisch in Grundschulen (mit insgesamt 650 Unterrichtsstunden) und in Mittelschulen als erste (im Umfang von 420 Unterrichtsstunden) oder zweite Fremdsprache als Wahlfach (im Umfang von 200 Unterrichtsstunden) unterrichtet. Im Hochschulbereich wird in die Studiengänge oft fachbezogener Englischunterricht integriert. Von den Studienanfängern wird erwartet, dass sie aufgrund der Sprachausbildung in der Grund- und Mittelschule über solide Allgemeinkenntnisse der englischen Sprache verfügen. Zavašnik (2006, S. 50-55) führt an, dass Hochschuleinrichtungen 
(HE) bei der Planung der Stundenzahl und Inhalte des Fachenglischunterrichts vollkommen autonom sind. In den 90er Jahren wurde an einigen HE der Fachenglischunterricht aus den Studiengängen gestrichen, was die Entwicklung der Fächer und Sprachkenntnisse der Studierenden eingrenzte. Wie bereits festgestellt, gibt es auf Nationalebene keine Absprache oder Fremdsprachenpolitik über den Fremdsprachenunterricht an den nichtphilologischen HE. Es wäre zu erwarten, dass das Ministerium für Hochschulwesen einen Ausschuss hat, der sich um den Spracherwerb der Studierenden in den international orientierten Studiengängen bemühen und die Richtlinien für die Planung, Bewertung und Normen nach den GeR-Kriterien ausarbeiten würde. Infolgedessen sind die Planung und Gestaltung der Fremdsprachenpolitik jeder einzelnen HE (sogar jedem einzelnen Fachsprachlehrer) überlassen, was keineswegs zu den einheitlichen Kriterien für die Bewertung des Eingangs- und Abschlussniveaus der Fremdsprachenkenntnisse beiträgt.

Das Eingangsniveau der Fremdsprachenkenntnisse nach der abgeschlossenen allgemeinen oder fachbezogenen Reifeprüfung kann nach den GeR-Kriterien nur grob eingeschätzł werden. So können die Kenntnisse der englischen und deutschen Sprache (als erster Fremdsprache) nach dem allgemeinen Abitur gemäß dem GeR mit B2 eingeschätz† werden, was den Ausgangspunkt für die Planung des Fachfremdsprachenunterrichts im Hochschulbereich darstellen könnte.

Deutsch wird immer öfter als zweite oder dritte Fremdsprache unterrichtet (GI Ljubliana, 2009), z.B. als zweite Fremdsprache in der Grundschule im Rahmen des dreijährigen Wahlfaches (mit 204 Unterrichtsstunden). So können nach der abgeschlossenen neuniährigen Grundschulausbildung die Kenntnisse der Schüler auf dem Sprachniveau zwischen A1 und A2 (GeR) eingeschätzt werden. Auch in der weiterführenden technischen oder berufsbezogenen Mittelschule, die vier Jahre dauert, bzw. im Gymnasium, können die Schüler Deutsch als zweite Fremdsprache lernen. Nach der abgeschlossenen Mittelschule werden die Deutschkenntnisse der Gymnasiasten nach 420 Stunden Unterricht zwischen B1 und B2 (GeR) bewertet. Die Deutschkenntnisse der Schüler, die die technische oder Berufsmittelschule besuchten, könnten wegen der geringeren Unterrichtszahl auf der Niveaustufe zwischen A1 und A2 (GeR) bewertet werden (Horvath, 2008, S. 381-382). 
Margit Horvath, Manica Danko, Polonca Kovač, Janez Stare

Fremdsprachenausbildung - Bedarfs- und Erfahrungsanalyse der Fremdsprachenausbildung in der slowenischen öffentlichen Verwaltung

Fremdsprachenprogramme an den einzelnen $\mathrm{HE}$ werden aufgrund der Erwartungen des Arbeitsmarktes oder der künftigen Arbeitgeber erstellt, wenn Ergebnisse der Bedarfsanalysen zu Fremdsprachen nach der abgeschlossenen Ausbildung vorliegen. Auch die Fakultät für Verwaltungswissenschaften hat keine ganzheitlich ausgearbeitete Bedarfsanalyse (zu Fremdsprachen), weil die Anstellung ihrer Absolventen sehr schwer vorauszusehen ist. Diese können sowohl im privaten wie auch im öffentlichen Sektor angestellt werden. Es ist fast unmöglich, den Bedarf an Fremdsprachen nach einzelnen Stellenbereichen genau zu untersuchen. Eine Lösung wäre, dass die Fremdsprachenlehrer die Studiengänge näher kennenlernen und mit Kollegen anderer Studienfächer in Verbindung treten. So könnten die Studenten während ihres Studiums Fremdsprachen besser erlernen und damit eine Basis für deren spätere Verwendung auf dem Arbeitsmarkt haben.

\section{Forschungsproblem}

Der Ausgangspunkt für die Forschung war die Frage nach dem Fremdsprachenbedarf (an englischer, deutscher und evtl. einer dritten Sprache) der Angestellten in der öffentlichen Verwaltung bezüglich ihrer Sprachkompetenzen (Schreiben, Sprechen, Hören und Lesen) am Arbeitsplatz. Die Forschungsergebnisse sollten die Basis für die Weiterentwicklung und Modernisierung des Fremdsprachenunterrichts (bzw. der erwähnten Fremdsprachen) an der Bachelor- und Master-Ebene (Entwicklung der Kompetenzen von Studierenden) sowie die Angebotsvorbereitung des Beratungs- und Ausbildungszentrums an der Fakultät für Verwaltungswissenschaften der Universität Ljubljana (bessere permanente Fortbildung der Angestellten in der slowenischen öffentlichen Verwaltung) sein.

\subsection{Vorangegangene Untersuchung}

Die vorangegangene Untersuchung des Forschungsproblems fand im Rahmen eines internen Forschungsprojektes der Fakultät für Verwaltungswissenschaften statt (Danko, Stare \& Horvath, 2008), in dem der Bedarf der Studierenden und Diplomanten der Fakultät für Verwaltungswissenschaften im Bereich der Fremdsprachenausbildung bei den Studienfächern Englisch und Deutsch analysiert wurde. Für die Untersuchung wurde eine Online-Umfrage entwickelt, mit deren Hilfe der 
Bedarf angestellter Diplomanten an der englischen und deutschen Sprache bzw. einer dritten Fremdsprache ermittelt und analysiert wurde.

Das Ziel des Forschungsprojektes war, aufgrund der Analyse der Sprachkenntnisse der Diplomanten der öffentlichen Verwaltung zu notwendigen Erkenntnissen zu kommen, die zur Erstellung von hochwertigen Lernmaterialien und kontinuierlicher Organisation der Weiterbildung auf dem Gebiet der Fachfremdsprache bei Verwaltungsangestellten beitragen würden. An der Umfrage nahmen 121 Befragte teil. Die meisten waren in der öffentlichen bzw. Staatsverwaltung, zum Teil aber auch im Privatsektor angestellt. Weniger als die Hälfte der Befragten belegte im Abitur auch eine Fremdsprache. Darunter studierte die Mehrheit der Diplomanten außerordentlich, d.h. sie absolvierte nur ein Drittel der Kontaktstunden, die für die Fremdsprache im Rahmen des ordentlichen Grundstudiums vorgesehen war.

Die Mehrheit der Befragten schloss die Mittelschulbildung mit gutem Durchschnitt ab. Ähnlich bewerteten sie auch ihre damaligen Englischund Deutschkenntnisse. Am besten schätzten sie ihre rezeptiven (Hören und Lesen) und schlechter ihre produktiven Fähigkeiten (Schreiben und Sprechen) ein. Laut ihren Angaben möchten sie ihre selbstständige mündliche Ausdrucks- und Verständigungsfähigkeit, das Schreiben, Hörverstehen und in geringerem Maße die Lesefähigkeit verbessern. Damals verwendeten nur wenige der Befragten Fremdsprachen am Arbeitsplatz; sie verständigten sich in größerem Maße mit den Sprechern, die keine Muttersprachler waren, nur mündlich. Nur ein unbeträchtlicher Teil der Befragten kommunizierte auch schriftlich. Sie lasen nur Fachliteratur oder Geschäftskorrespondenz, in geringerem Maße auch englische und deutsche Zeitungen.

Die Studie ergab, dass vor allem die Aktivitäten, wie das Schreiben auf dem Gebiet der Geschäftskorrespondenz, kurze Redeauftritte sowie Arbeit am Wortschatz als besonders sinnvoll bewertet wurden. Sie wurden als Hilfe bei Umgang und Verständigung mit den ausländischen Geschäftspartnern und als Folge einer immer größeren Rolle der (Fremd)Sprache im Geschäftsleben (bei Seminaren, Konferenzen, Kongressen) erwähnt.

Wegen der Berufsausübung und der Möglichkeit, das Studium im Ausland fortzusetzen, fanden die Befragten auch das Lesen der Fachzeitschriften und der Studienliteratur, wie auch die Kenntnis der Fachbegriffe 
Margit Horvath, Manica Danko, Polonca Kovač, Janez Stare

Fremdsprachenausbildung - Bedarfs- und Erfahrungsanalyse der Fremdsprachenausbildung in der slowenischen öffentlichen Verwaltung

aus dem Bereich der öffentlichen Verwaltung als nützlich. Zugleich konnte mehr als die Hälfte bei dieser Frage keine Entscheidung treffen, was dadurch zu erklären ist, dass sie über den Bedarf an Fremdsprachenkenntnissen nicht informiert worden sind oder an ihrem Arbeitsplatz keiner vorhanden gewesen ist.

Anhand der Umfrageergebnisse kamen wir zum Schluss, dass es sinnvoll wäre, fachbezogene Fortbildung für verschiedene Bereiche der öffentlichen Verwaltung zu organisieren und Sprachkompetenzen der Verwaltungsangestellten für den Arbeitsplatzbedarf zu entwickeln. Infolgedessen entstand auch der Bedarf nach einer Bedarfs- und Erfahrungsanalyse der Fremdsprachenausbildung der Angestellten im slowenischen öffentlichen Dienst.

\subsection{Forschungsaufgabe}

Die Eingliederung Sloweniens in die Europäische Union und ihre mehrsprachige Organisation schufen einen Entwicklungsrahmen, innerhalb dessen es in den letzten 10 Jahren zu vielen Neuerungen auf dem Gebiet der Fachsprache und Sprachwissenschaft in Slowenien, vor allem in Hinsicht auf das Übersetzen, Dolmetschen und die Terminologie kam (Erbič, 2008, S. 107). Die Eingliederung der Republik Slowenien in die EU und die Übersetzung ihrer Rechtsordnung in die Fremdsprachen sowie die daraus entstandenen Folgen (Zusammenarbeit der öffentlichen Verwaltung mit den Institutionen der EU und anderen Ländern bzw. ihren Vertretern, höhere Einwanderanzahl und Kommunikation mit ihnen) steigern die Bedeutung der Fremdsprachenausbildung in der öffentlichen Verwaltung.

Mit der Bedarfs- und Erfahrungsanalyse in der slowenischen öffentlichen Verwaltung wollten wir ermitteln, wie die EU-Mitgliedschaft Sloweniens und seine Einwanderungspolitik den Bedarf der Verwaltungsangestellten an den Fremdsprachenkenntnissen beeinflussten, wie Beschäftigte im öffentlichen Dienst bei der Ausübung amtlicher Geschäftstätigkeiten mit den ausländischen Kunden kommunizieren und in welchen Fremdsprachen sie sich verständigen.

Bei der Vorbereitung des Fragebogens gingen wir von den folgenden Hypothesen aus: 
- nach dem Beitritt Sloweniens zur EU nahm der Bedarf der Angestellten in der öffentlichen Verwaltung an Fremdsprachenkenntnissen zu;

- die Inhaltsbereiche, wo der Bedarf am Fremdsprachenerwerb am größten ist, hängen mit der Zusammenarbeit Sloweniens mit der EU zusammen;

- in der öffentlichen Verwaltung herrscht der Bedarf an Kenntnissen der englischen, deutschen und französischen Sprache vor;

- Fremdsprachen werden am meisten für auswärtige Angelegenheiten beim Ministerium benötigt;

- Fremdsprachenkenntnisse benötigen vor allem leitende Verwaltungsangestellte;

- Organisationen der öffentlichen Verwaltung finanzieren keine Fremdsprachenkurse;

- in den einzelnen statistischen Regionen und Organisationen der öffentlichen Verwaltung werden Fremdsprachenkenntnissen unterschiedlich benötigt;

- die Fort- und Weiterbildung der Angestellten im Fremdsprachenbereich ist nicht kontinuierlich, systematisch und einheitlich.

Mit der Forschung wollten wir ermitteln,

- ob Angestellte in der öffentlichen Verwaltung bei der Ausübung amtlicher Tätigkeiten Fremdsprachenkenntnisse benötigen;

- welche Fremdsprachenkenntnisse am meisten benötigt werden;

- in welchen Situationen die Fremdsprachen am häufigsten benötigt werden und wie oft sie angewandt werden;

- welche Fremdsprachkompetenzen besonders benötigt werden;

- für welche Inhaltsbereiche der Bedarf an Fremdsprachen am höchsten ist;

- ob sich Angestellte im öffentlichen Dienst im Bereich des Fremdsprachenerwerbs regelmäßig ausbilden;

- wer (wenn überhaupt) die Fremdsprachenkurse für die im öffentlichen Dienst beschäftigten Angestellten finanziert und

- in welchen Organisationen es den größten Bedarf an Fremdsprachenausbildung gibt. 


\section{Untersuchung der Fremdsprachenausbildung - Zustands- und Bedarfsaufnahme}

\subsection{Ablauf der Untersuchung}

Für den Bedarf der Untersuchung wurde ein Fragebogen für die Organisationen der öffentlichen Verwaltung in ganz Slowenien entwickelt. Er bestand aus 41 offenen und geschlossenen Fragestellungen, die sich auf die allgemeinen Informationen über die Organisationen, Angaben über den Bedarf der öffentlichen Angestellten an Fremdsprachenkenntnissen bei der Ausführung ihrer Arbeitsaufgaben und über die Durchführung und Finanzierung der Fremdsprachenausbildung seitens der Organisationen sowie den Einfluss der Fremdsprachenkenntnissen auf die Qualität ihrer Dienstleistungen. Vor der Durchführung der Untersuchung gab es eine "Pilotuntersuchung", in die eine Verwaltungsbehörde und eine Gemeindeverwaltung eingeschlossen wurden. Anhand dieser Ergebnisse wurde der Fragebogen zweckgemäß erstellt.

Der Fragebogen wurde so gestaltet, dass alle Bereiche der öffentlichen Verwaltung, d.h. 441 Organisationen der öffentlichen Verwaltung einbezogen wurden. Der Fragebogen wurde an Ministerien (15), den Ministerien unterstellte Behörden (40), Verwaltungsbehörden (58), Regierungsdienste (16), Gemeindeverwaltungen (210) und Träger von öffentlichen Befugnissen (d.h. Renten- und Invaliditätsversicherungsanstalt ${ }^{9}$ (9), Krankenversicherungsanstalt Sloweniens ${ }^{10}$ (10), Arbeitsamt (12), Unterrichtsbehörde der Republik Slowenien "1 (9) und Zentren für Sozialarbeit (62)) verschickt.

Aufgrund der Kosten- und Zeitanalyse haben wir uns für die OnlineBefragung entschieden. Hinsichtlich der Mittel, die zur Verfügung standen, wäre keine andere Befragungsform (z.B. per Post oder persönliche Befragung ${ }^{12}$ ) sinnvoll gewesen. Obwohl uns bewusst war, dass die Teilnahme bei Online-Umfragen geringer ist, schätzten wir ein, dass zwischen 10 und 30 Prozent der Befragten den ausgefüllten Fragebogen zurücksenden werden. Zugleich sahen wir voraus, dass so ein Ergebnis

\footnotetext{
9 ZPIZ (Zavod za pokojninsko invalidsko zavarovanje)

10 ZZZS (Zavod za zdravstveno zavarovanje Slovenije)

11 Zavod za šolstvo

12 ein Face-to-Face Interview
} 
repräsentativ für die einzelnen Regionen, Segmente oder Organisationen in der öffentlichen Verwaltung stehen kann.

Wir haben ein gutes Drittel von zufällig ausgewählten Befragten angerufen, ihnen die Absicht und das Ziel des Projektes vorgestellt und sie zur Teilnahme an der Umfrage eingeladen. Der Link zum OnlineFragebogen wurde per Post verschickt. Die Befragung daverte zwei Monate (vom 13. Januar bis zum 13. März 2009) und in dieser Zeit wurden 103 Online-Fragebögen (23,35\%) ausgefüllt. Wir stellen fest, dass die Befragung (bezüglich der Anzahl aller Organisationen im öffentlichen Sektor) zwar klein, aber vielfältig war, und deshalb eine sinnvolle Darstellung von Trends bei der Fremdsprachenausbildung in einem größeren Teil des öffentlichen Sektors ermöglicht.

Die Angaben über alle Befragten wurden im ersten Teil des Fragebogens gesammelt. Im zweiten Teil sind unterschiedliche Fragen. Z.B. die Frage zu Inhaltsbereichen, wo der größte Bedarf an Erwerb der Fremdfachsprache ist, beantworteten alle Befragten (103). Auf die Fragen über den Bedarf an Fremdsprachenkenntnissen und über einige allgemeine Daten bezüglich der Fremdsprachenausbildung erhielten wir $15,19 \%$ (67) Antworten, auf die Fragen über den Ablauf der Fremdsprachenausbildung 4,67\% (21) Antworten. Im dritten Teil des Fragebogens bekamen wir keine Antworten von fast einem Drittel der Befragten. Zum Beispiel antworteten auf die Frage über die Finanzierung der Fremdsprachenausbildung nur 14,51\% (64) und über die Gründe für den Bedarf an der Fremdsprachenausbildung 15,19\% (67) der Befragten. Aus diesem Grund sind in der detaillierten Datenanalyse drei unterschiedliche Zahlen der gesammelten Antworten zu finden.

Die Online-Umfrage wurde von Angestellten an verschiedenen Arbeitsplätzen ausgefüllt. Unter ihnen gibt es am meisten Oberberater (27\%), Leiter (17\%), Direktoren (14\%) und Berater (1 1\%) für verschiedene Bereiche, sowie 5\% Sekretärinnen und 4\% Untersekretäre. Der Vergleich zwischen der ersten und zweiten Arbeitsaufgabe dieser Personen weist keinen großen Unterschied auf. Die meisten sind für die Führung und Organisation der Arbeit zuständig (z.B. im Rahmen der ersten Aufgabe $27,18 \%$ und der zweiten 10,67\%). 10,67\% der Angestellten üben Personalangelegenheiten aus, 9,70\% koordiniert und beaufsichtigt die Arbeit der Mitarbeiter, 5,83\% sorgt für die Ausbildung und 4,85\% für die internationale Zusammenarbeit. 
Margit Horvath, Manica Danko, Polonca Kovač, Janez Stare

Fremdsprachenausbildung - Bedarfs- und Erfahrungsanalyse der Fremdsprachenausbildung in der slowenischen öffentlichen Verwaltung

Eine gute Hälfte aller befragten Organisationen hat weniger als 50 Angestellte. Die meisten Organisationen $(21,35 \%)$ haben zwischen 20 und 30 Angestellte; 16,5\% zwischen 10 und 20; 7,76\% zwischen 30 und 40 und $3,88 \%$ zwischen 40 und 50 Angestellte.

Nach der abgeschlossenen Online-Umfrage wurden die Ergebnisse zuerst im Excel ausgewertet. Da sich das Programm für zusätzliche Korrelationsberechnungen zwischen verschiedenen Fragen als ungeeignet erwies, wurde eine weitere Datenauswertung mit dem statistischen Programm SPSS ${ }^{13}$ durchgeführt. Trotz einer relativ niedrigen Anzahl der Befragten (23,35\%), die sich an der Befragung aktiv beteiligt haben, ist im Ergebnis das Bild der Fremdsprachenpolitik im größten Teil der öffentlichen Verwaltung und des durchschnittlichen Bedarfs an Fremdsprachenkenntnissen bei den meisten Verwaltungsangestellten enthalten. Obwohl die Zahl derienigen, die den Fragebogen ausgefüllt haben, niedriger als 30 Prozent ist, kann daraus der Schluss auf die Situation und den Bedarf im Bereich der Organisation und Durchführung von Fremdsprachenkursen im größten Teil des öffentlichen Sektors in Slowenien gezogen werden. Es ist aber auch wahr, dass die recht hohe Nichtbeteiligung an der Untersuchung (76,65\%) keinen glaubwürdigen Repräsentationsschluss darstellen kann. Einige statistische Regionen oder Organisationen, die verhältnismäßig klein sind (z.B. nur eine Außenstelle der Krankenversicherungsanstalt Sloweniens sandte den ausgefüllten Fragebogen zurück) haben nur einen ausgefüllten Fragebogen oder gar keinen (z.B. von der Renten- und Invaliditätsversicherungsanstalt sowie dem Arbeitsamt kam kein Fragebogen zurück) zurückgesandt.

\subsection{Zusammenfassung der wesentlichen Erkenntnisse}

Die Untersuchung ergab, dass Angestellte in der öffentlichen Verwaltung bei ihrer Arbeit Englisch- (83\%) und Deutschkenntnisse (68\%) benötigen, außer auf den Gemeindegebieten mit der autochthonen italienischen und ungarischen Volksgruppe (Šturm et al., 2011) $)^{14}$. Der Bedarf an Kenntnissen

13 Abkürzung für die Bezeichnung Statistical Package for the Social Sciences

14 Die Prozedurregeln über den Sprachgebrauch im Verfahren sind aus den Verfassungsbestimmungen über die Amtssprache (Artikel 11 der Verfassung der Republik Slowenien) und dem Recht auf den Gebrauch der eigenen Sprache und Schrift abgeleitet (Artikel 62). Darum wird das Verwaltungsverfahren in der slowenischen Sprache geführt, jedoch auf den Gemeindegebieten mit der italienischen und ungarischen Minderheit, auch in der italienischen und ungarischen Sprache, falls in einer dieser Sprachen der Antrag eingereicht wurde bzw. der Kunde Italienisch oder Ungarisch spricht. Darum werden 
der französischen Sprache ist trotz der engeren internationalen Zusammenarbeit slowenischer öffentlicher Dienste mit den EUInstitutionen um 26,22\% niedriger als an den Kenntnissen der kroatischen oder serbischen Sprache, welche die Angestellten in der öffentlichen Verwaltung wegen der Einwanderung der Staatsbürger aus den Nachfolgestaaten des ehemaligen Jugoslawien in guter Hälfte von Organisationen benötigen.

\section{Diagramm 1: Bedarf der Angestellten in der öffentlichen Verwaltung an Fremdsprachenkenntnissen}

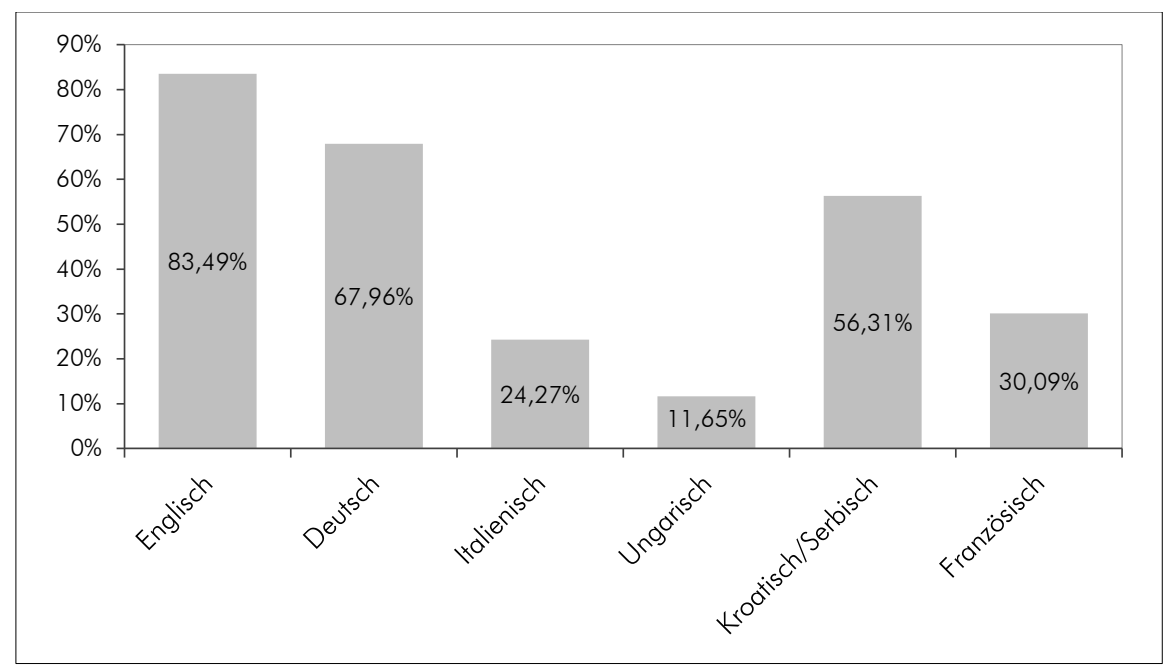

Quelle: Umfrage, 2009

Der Bedarf der Angestellten im öffentlichen Dienst an Englisch- und Deutschkenntnissen unterscheidet sich im Umfang. In den Organisationen bis zu 10 Angestellten $(39,80 \%$ oder 41$)$ ist der Bedarf an Deutsch-

Anträge auch außerhalb des zweisprachigen Gebietes (siehe Absatz 5, Artikel 65, Gesetz über das Verwaltungsverfahren) in der slowenischen, italienischen und ungarischen Sprache gestellt, obwohl die Behörden ihre Tätigkeiten außerhalb dieses Gebietes ausschließlich in der slowenischen Sprache ausüben. Den Angehörigen der Minderheiten müssen sie den Rechtsanspruch gewährleisten, indem sie auf Kosten der Behörde für die Übersetzung der Anträge sorgen und die Teilnahme am Verfahren im Falle der mündlichen Behandlung durch einen Dolmetscher ermöglichen. Gemäß den Vorschriften über die Amtssprache wird allen, die die Amtssprache nicht können, das Recht auf die Verfolgung des Verfahrens mithilfe eines Dolmetschers garantiert. Die Behörde ist nicht verpflichtet amtliches Schriftstück zu übersetzen, es sei denn das steht im Regionalgesetz fest, z.B. im Ausländergesetz aus dem Jahr 2011, laut dem Ausländern der Bescheid über die Heimkehr ausgehändigt wird, in dem der Strafspruch und die Rechtsbelehrung mindestens in fünf Sprachen übersetzt werden müssen (Kovač et al., 2012). 
kenntnissen höher als an Kenntnissen der englischen Sprache $(25,24 \%$ oder 26). Es geht um die befragten Organisationen der öffentlichen Verwaltung, wie kleinere Gemeinden und Verwaltungsbehörden aus den statistischen Regionen an der österreichischen Grenze: Draugegend ${ }^{15}$ $(14,60 \%)$, Oberkrain ${ }^{16}(12,60 \%)$ und Unterkärnten ${ }^{17}(7 \%)$. Bei Organisationen mit über 30 Angestellten (z.B. Ministerien und den Ministerien nachgeordneten Behörden) ist der Bedarf an Englisch (14\%) um die Hälffe höher als an Deutschkenntnissen (7\%). In den Organisationen zwischen 10 und 20 bzw. 20 und 30 Angestellten gibt es keinen größeren Unterschied im Bedarf an beiden Sprachen (10,67\% Englisch, 9,70\% Deutsch).

Diagramm 2: Bedarf an Englisch- und Deutschkenntnissen

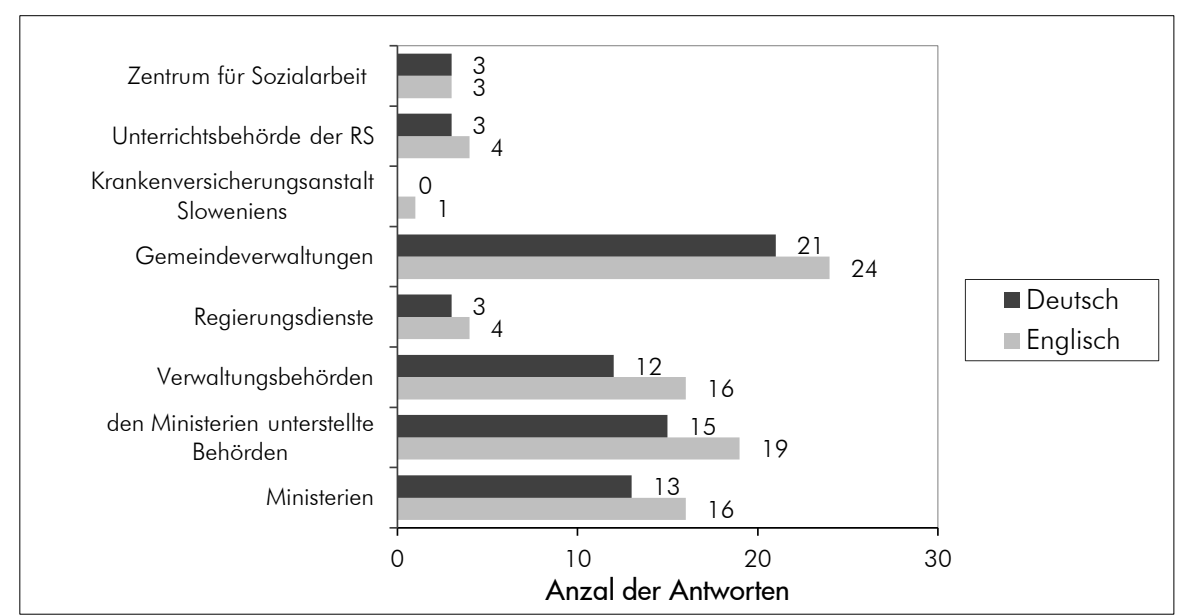

Quelle: Umfrage, 2009

Den Bedarf an Englisch- bzw. Deutschkenntnissen zeigen am häufigsten Angestellte bei den Ministerien unterstellten Behörden (100\% Englisch und 78,94\% Deutsch), Ministerien (94,11\% Englisch und 76,47\% Deutsch), Gemeindeverwaltungen (85,71\% Englisch und 75\% Deutsch) und Verwaltungsbehörden (80\% Englisch und 60\% Deutsch). Der geringste Bedarf besteht bei dem Zentrum für Sozialarbeit und der Krankenversicherungsanstalt Sloweniens.

15 Podravska

16 Gorenjska

17 Koroška 
Vom Gesichtspunkt der Untersuchung des Fremdsprachenbedarfs wurde festgestellt, dass Fremdsprachenkenntnisse nicht nur leitende Angestellte benötigen, sondern auch Oberberater, (Staats)Sekretäre, Angestellte im öffentlichen Dienst die mit Ausländern arbeiten (vgl. Beamte für das Arbeitsgebiet Ausländer) sowie Angestellte im Bereich der internationalen Zusammenarbeit.

\section{Diagramm 3: Bedarf an Englisch- und Deutschkenntnissen bezüglich der Arbeitsstellung}

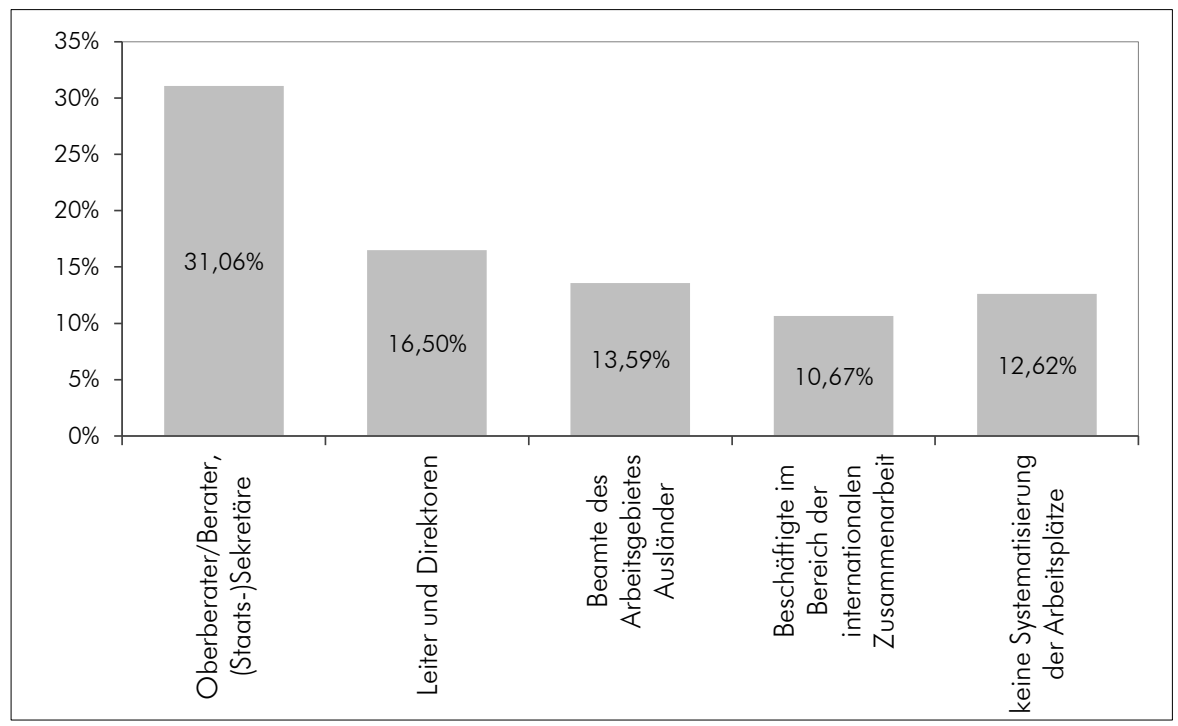

Quelle: Umfrage, 2009

Mit der Datenauswertung konnte nicht ermittelt werden, ob sich der Bedarf der Angestellten im öffentlichen Dienst an Fremdsprachenkenntnissen nach dem EU-Beitritt Sloweniens tatsächlich erhöhte. Es wurde festgestellt, dass sich Angestellte zwischen 2006 und 2007 im Fremdsprachenbereich um ein Drittel mehr fort- und weiterbildeten als in der Zeit von Anfang 2008 bis Mitte 2009. Nach Vorhersagen müsste sich Ende 2010 diese Zahl verdoppeln, was angesichts der Wirtschafts- und Finanzsituation sehr fraglich ist.

Bedeutend ist auch die Tatsache, dass die Hälfte der Befragten (44 von 103 oder 42,71\%) die Frage nach dem Bedarf in den einzelnen Inhaltsbereichen nicht beantwortet hat. Diejenigen, die darauf eingingen (57,25\%), führten verschiedene Bereiche an, die wegen einer einfachen Interpretation in thematische Gruppen verteilt wurden: EU-Ausschreibungen, EU-Gesetzgebung, internationale Zusammenarbeit, Rechtssprache, 
Margit Horvath, Manica Danko, Polonca Kovač, Janez Stare

Fremdsprachenausbildung - Bedarfs- und Erfahrungsanalyse der Fremdsprachenausbildung in der slowenischen öffentlichen Verwaltung

Ausstellung von Bescheiden Ausländern (z.B. im Zusammenhang mit der Staatsbürgerschaft), allgemeine Kommunikation mit Ausländern, Protokoll und Fremdenverkehr. Die Untersuchung ergab, dass die Beschäftigten in der öffentlichen Verwaltung die Fremdsprachen am häufigsten dann verwenden, wenn sie an EU-Ausschreibungen teilnehmen (30,5\%), europäische Gesetzgebung verfolgen (25,42\%), sich an der internationalen Zusammenarbeit beteiligen (16,94\%), und internationale Arbeitsgruppen führen oder mit benachbarten Gemeinden kommunizieren.

\section{Diagramm 4: Fremdsprachenbedarf bezüglich des Inhalts von Arbeitsbereichen (wöchentlich)}

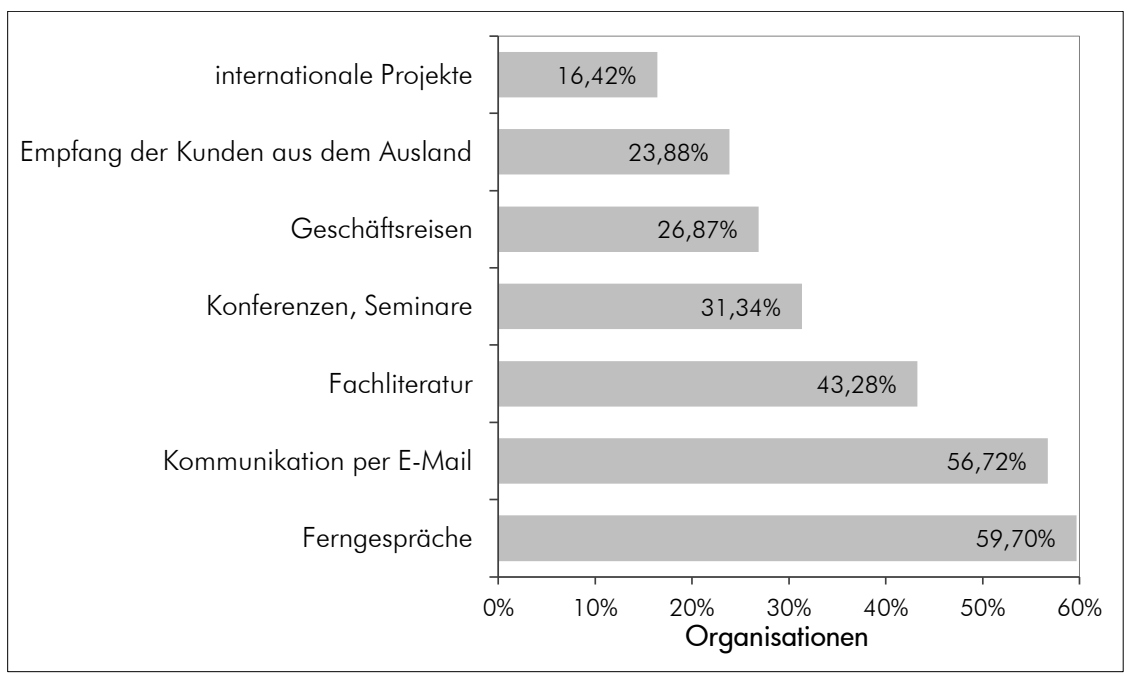

Quelle: Umfrage, 2009

Aus den Ergebnissen der Befragung geht hervor, dass die Angestellten im öffentlichen Dienst häufiger Fremdsprachenkenntnisse aus dem Rechtsbereich (15,25\%; vergleiche z.B. für Italienisch und Ungarisch Šturm et al., 2011) und bei der Kommunikation mit den ausländischen Kunden benötigen, z.B. bei der Ausstellung der Bescheide über den Erwerb der Staatsangehörigkeit oder Anschaffung von Immobilien (13,55\%). Außerdem werden sie noch im Bereich von Protokoll, Fremdenverkehr, Steuern und Fachentwicklung gebraucht. Im Rahmen der Geschäftskommunikation wird die Fremdsprache dann verwendet, wenn Ferngespräche mit dem Ausland geführt (59,70\%) und E-Mails geschrieben werden $(56,72 \%)$ sowie Fachliteratur gelesen wird $(43,28 \%)$. 
Margit Horvath, Manica Danko, Polonca Kovač, Janez Stare

Fremdsprachenausbildung - Bedarfs- und Erfahrungsanalyse der

Fremdsprachenausbildung in der slowenischen öffentlichen Verwaltung

\section{Diagramm 5: Wer ermöglicht den Angestellten die Fremdsprachenausbildung?}

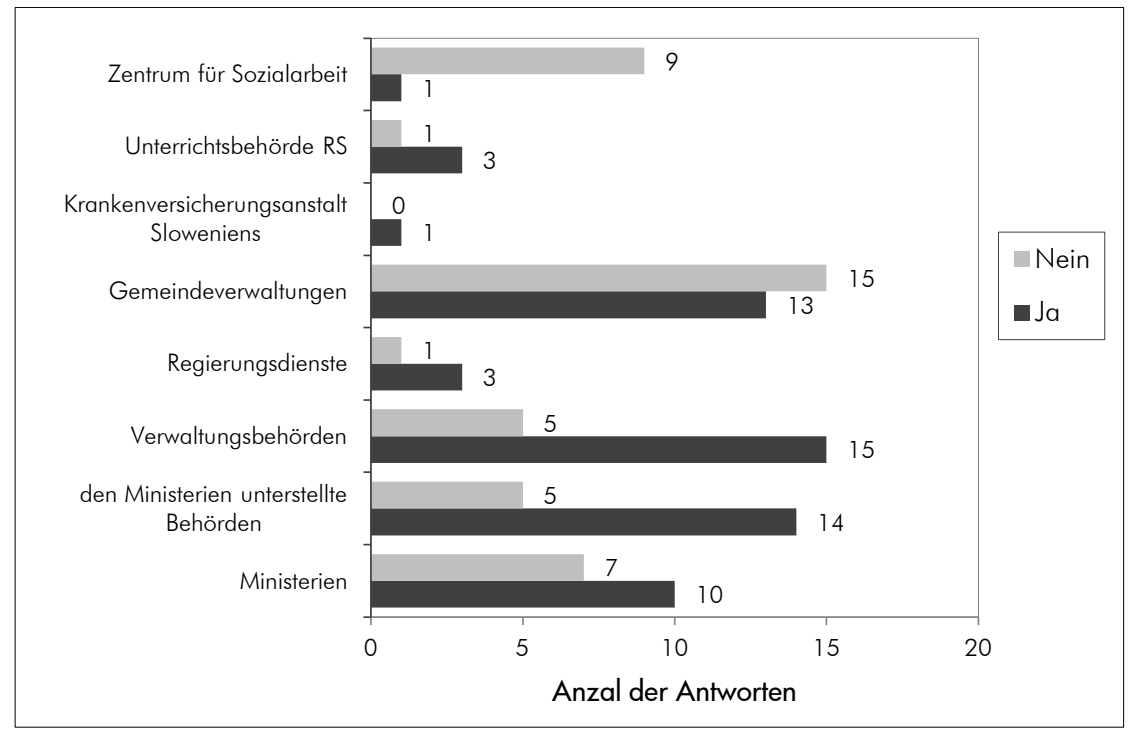

Quelle: Umfrage, 2009

Nach den Analyseergebnissen ermöglicht fast die Hälfte der Befragten (60 bzw. 58,3\%) den Beschäftigten die Fort- und Weiterbildung im Fremdsprachenbereich. Die höchste Zahl liegt bei den Verwaltungsbehörden (15 bzw. 75\%), den Ministerien unterstellten Behörden (14 bzw. 73,68\%) und Ministerien (10 bzw. 66,66\%). Obwohl die Ergebnisse ergaben (siehe Diagramm 2), dass die Beschäftigten in den Gemeindeverwaltungen bei ihrer Arbeit am häufigsten Englisch- (85,71\%) und Deutschkenntnisse (75\%) benötigen, ermöglicht mehr als die Hälffe der befragten Gemeinden (15 bzw. 53,57\%) ihren Angestellten keine Fremdsprachenausbildung. 


\section{Diagramm 6: Bedarfsermittlung an der Fremdsprachenausbildung}

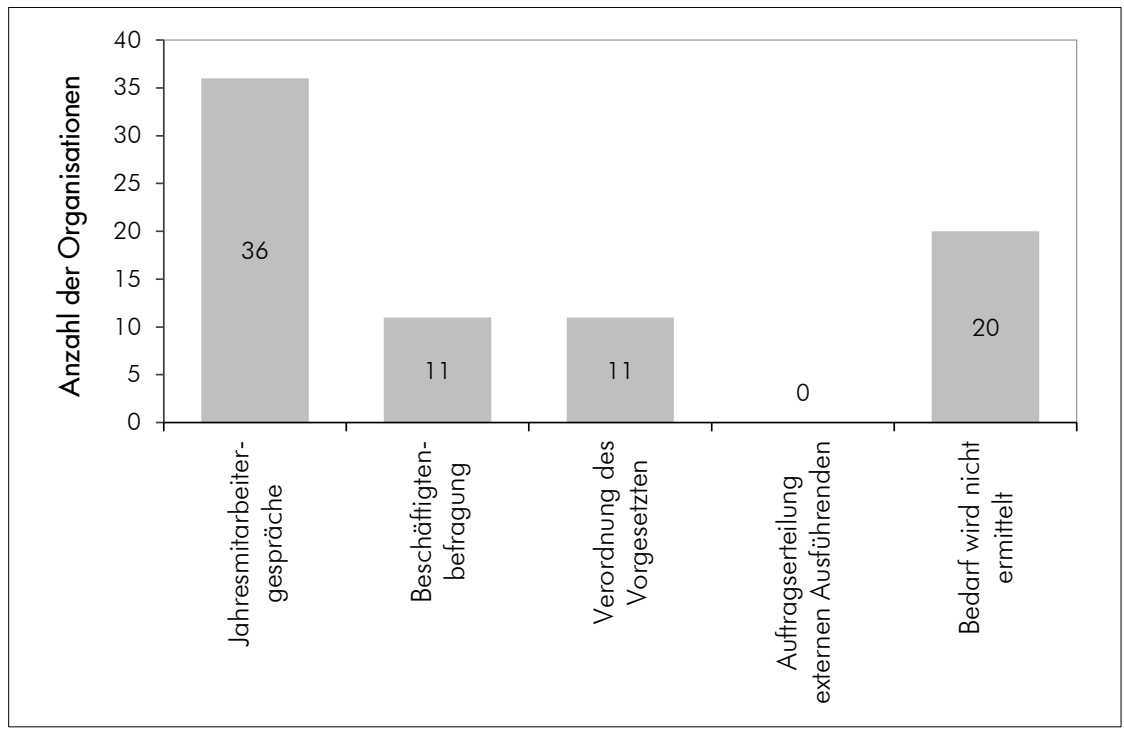

Quelle: Umfrage, 2009

Informationen über die Fremdsprachenweiterbildung der Angestellten in der öffentlichen Verwaltung sammelt nur gut die Hälfte der Befragten. Die Mehrheit ermittelt den Bedarf der Mitarbeiter mithilfe der Jahresmitarbeitergespräche (36 bzw. 53,73\%). Ein geringerer Anteil der Organisationen (11 bzw. 16\%) stellt die Bedürfnisse der Beschäftigten nach den Fremdsprachenkenntnissen aufgrund Fragebögen oder gemäß der Verordnung der Vorgesetzten fest. 30\% der Organisationen in der öffentlichen Verwaltung (20), die auf die Frage antworteten, stellt diese Bedürfnisse nicht fest.

Die Planung der Fremdsprachenausbildung der Angestellten in der öffentlichen Verwaltung (Diagramm 7) wird am meisten durch die Anforderungsstufe des Arbeitsplatzes (95,24\%) und ihre Wünsche sowie von der Bereitschaft zur Fremdsprachenbildung (90,48\%) beeinflusst. Einen wichtigen Faktor bei der Entscheidung über die Durchführung des Fremdsprachenkurses für Angestellte stellen auch Finanzmittel dar $(85,71 \%)$. Fremdsprachenkurse für Angestellte werden auch, allerdings seltener, aufgrund der Ausbildungspolitik der Organisation und Verordnung des Vorgesetzten durchgeführt. 
Margit Horvath, Manica Danko, Polonca Kovač, Janez Stare

Fremdsprachenausbildung - Bedarfs- und Erfahrungsanalyse der

Fremdsprachenausbildung in der slowenischen öffentlichen Verwaltung

\section{Diagramm 7: Planung der Fremdsprachenausbildung in der öffentlichen Verwaltung}

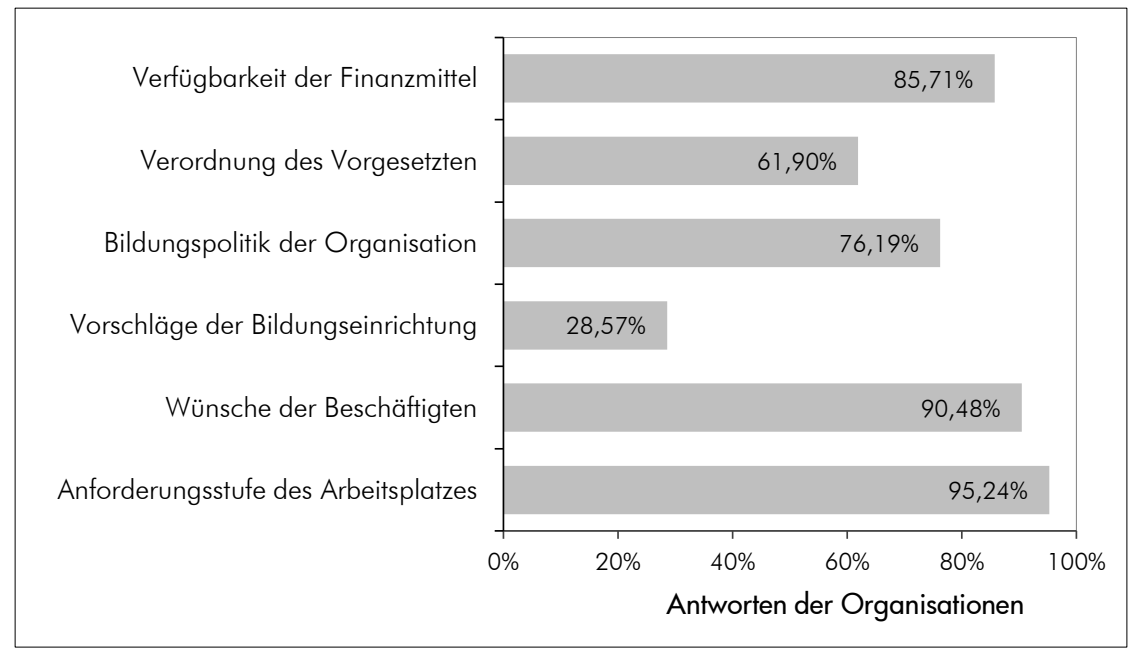

Quelle: Umfrage, 2009

Die Mehrheit der Organisationen in der öffentlichen Verwaltung, die den Angestellten Fremdsprachenausbildung ermöglichen (80,95\%), deckt alleine die gesamten Kosten für die Kurse. Weniger als 10 Prozent der Organisationen erstatten nur einen Teil der Kosten zurück, während der gleiche Anteil von Organisationen die Kosten der Fremdsprachenausbildung nicht zahlt. Die Mehrheit der Befragten antwortete, dass sich der Bedarf an Fremdsprachen und der Fremdsprachenausbildung im öffentlichen Sektor erhöht. 27\% der Organisationen sind der Meinung, dass sich der Bedarf verändert, während 3,2\% der Organisationen davon überzeugt sind, dass er zurückgeht.

Bei den Angestellten im öffentlichen Dienst, die sich im Fremdsprachenbereich weiterbilden, sind vor allem Kurse der Allgemeinsprache (verschiedener Stufen) und die Konversation angefragt. Spezialisierte Fremdsprachenkurse benötigt man zur Aneignung der Amtssprache, Fachterminologie aus dem Bereich der Stevern, EU-Vorschriften, Fonds und Ausschreibungen, Rechtsterminologie, Fachbegriffe aus dem Bereich der Arbeit, Beschäftigung, Sicherheit und Gesundheit bei der Arbeit, Verhandlungen und Vorbereitungen auf die Ausschreibungen sowie der Arbeit mit ausländischen Kunden, z.B. bei der Regelung der standesamtlichen Angelegenheiten, Ausstellung der Führerscheine und Fahrzeugzulassungen - am meisten in der englischen $(42,86 \%)$ und 
deutschen Sprache $(23,81 \%)$ sowie anderen Sprachen wie Ungarisch, Kroatisch und Serbisch (19,04\%).

Nur ein Drittel der befragten Organisationen in der öffentlichen Verwaltung (67 bzw. 34,30\%) organisiert für die Angestellten Fremdsprachenkurse, die in den meisten Organisationen während der Arbeitszeit stattfinden (71,4\%). Fremdsprachenkurse außerhalb der Arbeitszeit veranstalten $28,6 \%$ der Organisationen.

\section{Diagramm 8: Fremdsprachenausbildung der Angestellten}

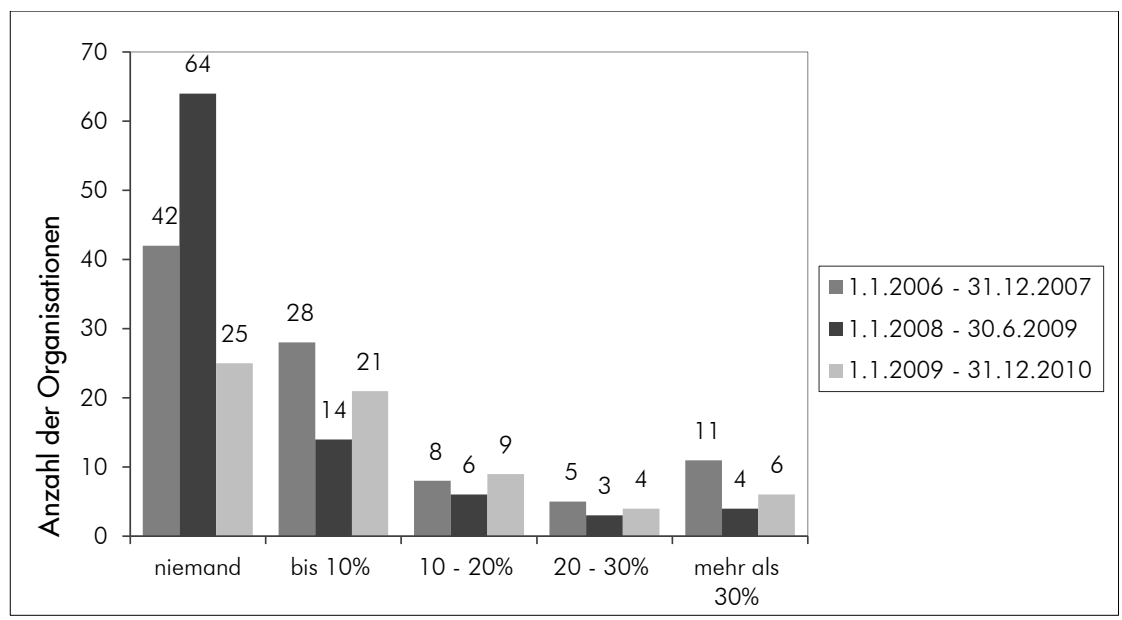

Quelle: Umfrage, 2009

Bei der Antwort auf die Frage, wie viele Angestellte in der Fremdsprachenausbildung sind, fallen beträchtliche Abweichungen zwischen den einzelnen Zeitabschnitten auf. Unter den Befragen sind am häufigsten Organisationen, die bis zu 10\% ihrer Angestellten in die Fremdsprachenausbildung einschließen. Von Anfang 2006 bis Ende 2007 gab es im Vergleich von Anfang 2008 bis Mitte 2009 um ein Drittel mehr Organisationen, die ihren Angestellten die Fremdsprachenweiterbildung ermöglichten. Die Gründe dafür könnten in den intensiveren Vorbereitungen Sloweniens auf die EU-Ratspräsidentschaft (von 1.1. bis 30.6.2008) liegen.

Festgestellt wurde auch, dass die Durchschnittszahl der Fremdsprachenunterrichtstunden von Anfang 2006 bis Ende 200741 Stunden pro Mitarbeiter war. Die Vorhersagen der Befragten bezüglich der Fremdsprachenausbildung ihrer Angestellten bis Ende 2010 waren 
ziemlich optimistisch ${ }^{18}$, obwohl es wegen der Vertiefung der Wirtschaftsund Finanzkrise, die sich auch auf den öffentlichen Sektor erstreckte, davon noch weniger gab.

Von 21 Befragten, die Fremdsprachenkurse für ihre Angestellten veranstalten (siehe Diagramm 5), antwortete die Mehrheit (76,20 \%), dass diese in Gruppen mit mehr als 8 Teilnehmern stattfinden. 4 Organisationen (19\%) haben kleinere Gruppen bis 6 Teilnehmern. Über Gruppen mit drei und weniger Teilnehmern berichteten nur zwei Organisationen (9,5\%). Es gab keine Angaben über individuelle Kurse, obwohl man erwartete, dass für die leitenden Angestellten (z.B. Leiter und Direktoren) maßgeschneiderte Kurse durchgeführt werden, die inhaltlich und zeitlich an ihre Arbeit angepasst sind.

In den meisten Organisationen (16 bzw. 76,19\%) besuchen Angestellte Fremdsprachenkurse, die mehrere Monate pro Jahr mit weniger Wochenstunden davern. 19,04\% bzw. 4 Organisationen organisieren Intensivsprachkurse für ihre Angestellten, die über einen längeren Zeitabschnitt mehrere Unterrichtsstunden täglich davern. Über ein- oder zweitägige Workshops berichtete nur eine Organisation.

\section{Diagramm 9: Formen der Fremdsprachenausbildung}

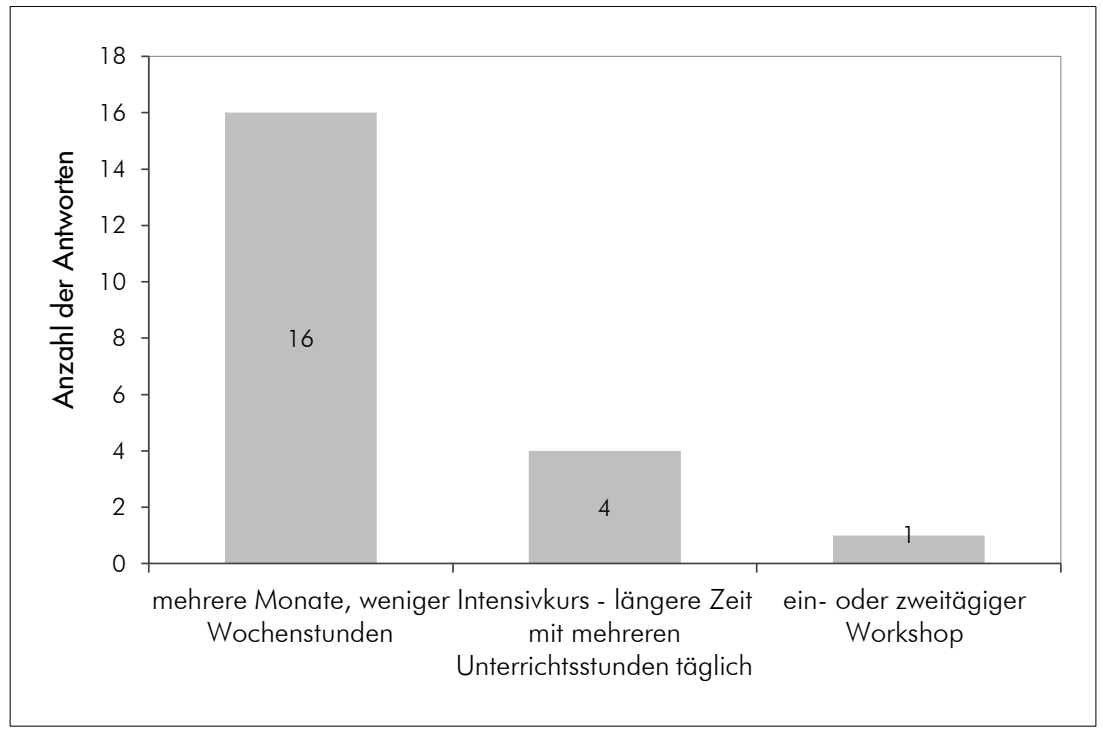

Quelle: Umfrage, 2009

18 Durchschnittliche Stundenzahl soll auf 46 steigen.

134 Uprava, letnik X, 1/2012 
Von 64 befragten Organisationen (62,13\%), die die Frage nach dem Zusammenhang zwischen dem Bedarf an Fremdsprachenkenntnissen und der Qualitätssteigerung der Verwaltungsverfahren beantworteten, vertritt die Mehrheit $(78,12 \%)$ die Meinung, dass die Fremdsprachenkenntnisse eine wichtige Rolle bei der Qualitätsverbesserung der Dienstleistungen spielen. Die restlichen 21,87\% Organisationen meinen, dass die Qualität der Dienstleistungen nicht auf die Fremdsprachenkenntnisse ihrer Angestellten zurückzuführen sind. Die Mehrheit unter ihnen gab an (vgl. Jerovšek \& Kovač, 2010), dass die Fremdsprachenkenntnisse für die meisten Arbeitsplätze in der öffentlichen Verwaltung nicht erforderlich sind.

\section{Diagramm 10: Gründe für die Steigerung des Fremdsprachenbedarfs}

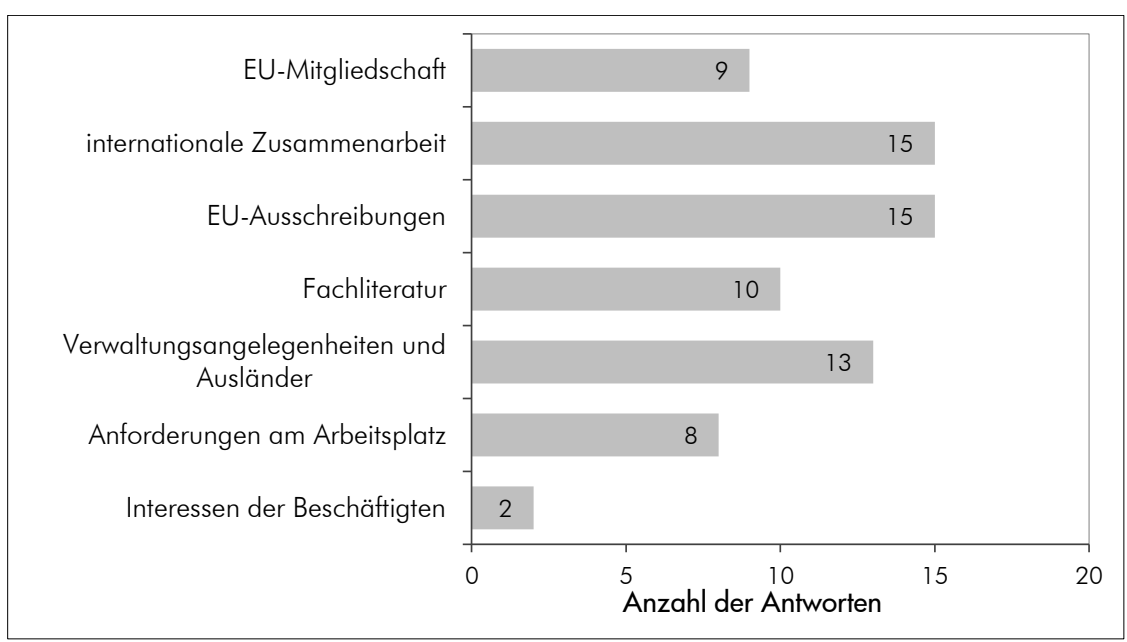

Quelle: Umfrage, 2009

Unter 67 Befragten ist die Mehrheit davon überzeugt, dass der Hauptgrund für die Bedarfssteigerung an Fremdsprachenkenntnissen die internationale Zusammenarbeit und die EU-Ausschreibungen sind. Zu den wichtigen Gründen zählen auch die Verwaltungsdienstleistungen und Arbeit mit Ausländern, die nach Slowenien eingewandert sind. 10\% der Befragten sind der Meinung, dass einer der Gründe für die Bedarfserhöhung an der Fremdsprachenausbildung auch darin liegt, dass sie ausländische Fachliteratur lesen müssen.

\section{5 Überprüfung der Hypothesen}

Durch die Untersuchung auf dem Bereich der Fremdsprachenausbildung der öffentlichen Angestellten im slowenischen Raum wurde nur eine 
Hypothese im Ganzen bestätigt. Weil die Zahl derjenigen, die an der Umfrage teilgenommen haben, zu niedrig ist, konnte eine Hypothese nicht überprüft werden. Es geht um die Hypothese, die sich auf den Unterschied bezüglich des Bedarfs an Fremdsprachenkenntnissen in der öffentlichen Verwaltung zwischen den einzelnen statistischen Regionen und Organisationen bezieht. Der Vergleich war leider nicht möglich, weil drei Anstalten am Befragungsmuster (Krankenversicherungsanstalt Slowenien, Unterrichtsbehörde der Republik Slowenien und Zentren für Sozialarbeit) mit zu geringer Zahl bzw. zwei (Renten- und Invaliditätsversicherungsanstalt und Arbeitsamt der Republik Sloweniens) gar nicht teilgenommen haben.

Die Untersuchung unterstützte zum Teil die Hypothese, dass die Fremdsprachenkenntnisse (Englisch und Deutsch) beim Ministerium vor allem für auswärtige Angelegenheiten gebraucht werden. Die Kenntnisse der beiden Sprachen benötigen Angestellte in fast allen Ministerien und den Ministerien unterstellten Behörden sowie in Gemeindeverwaltungen und Verwaltungsbehörden (vor allem in den statistischen Regionen an der österreichischen Grenze).

Die Untersuchung bestätigte die Hypothese, dass öffentliche Angestellte Bedarf an Englisch- (83\%) bzw. Deutschkenntnissen (68\%), sowie an der kroatischen bzw. serbischen Sprache (wegen der Einwanderung der Staatsbürger aus dem Gebiet des ehemaligen Jugoslawien) haben. Fremdsprachenkenntnisse benötigen nicht nur leitende Angestellte, sondern auch Berater, Oberberater, Sekretäre und Staatssekretäre, Angestellte im öffentlichen Dienst, die mit Ausländern arbeiten, und Angestellte im Bereich der internationalen Zusammenarbeit. Anhand der Untersuchung konnte nicht festgestellt werden, ob der Bedarf der öffentlichen Angestellten an Fremdsprachenkenntnissen nach dem Beitritt Sloweniens der EU tatsächlich zugenommen hat.

Die Untersuchung bestätigte (auch) die Hypothese, dass der Bedarf am Fremdsprachenerwerb am meisten mit der Zusammenarbeit zwischen Slowenien und der EU verbunden ist. Angestellte der öffentlichen Verwaltung benötigen Fremdsprachenkenntnisse vor allem wegen der Bewerbungen im Rahmen der EU-Ausschreibungen, der Verfolgung der europäischen Gesetzgebung und der internationalen Zusammenarbeit (z.B. bei der Führung internationaler Arbeitsgruppen oder bei der Kommunikation mit benachbarten Gemeinden). Darüber hinaus benötigen 
Margit Horvath, Manica Danko, Polonca Kovač, Janez Stare

Fremdsprachenausbildung - Bedarfs- und Erfahrungsanalyse der Fremdsprachenausbildung in der slowenischen öffentlichen Verwaltung

öffentliche Angestellte die Fremdsprache auch in anderen Situationen, jedoch seltener: z.B. im Bereich der Rechtssprache, Stevern und Fachentwicklung, bei Protokollen oder bei der Kommunikation mit ausländischen Kunden (wenn es sich um die Ausstellung der Bescheide hinsichtlich des Staatsangehörigkeitserwerbs oder der Immobilienanschaffung handelt) und im Fremdenverkehr.

Die Untersuchungsergebnisse bestätigten im Ganzen die Hypothese, dass die Weiter- und Fortbildung der öffentlichen Angestellten im Fremdsprachenbereich nicht kontinuierlich, systematisch und für alle gleich ist. Fast die Hälfte der befragten Organisationen der öffentlichen Verwaltung ermöglicht ihren Angestellten gar keine Fremdsprachenausbildung, während nur ein Drittel für sie Fremdsprachenkurse veranstaltet. Die Planung der Fremdsprachenausbildung für die Angestellten hängt in erster Linie von dem Arbeitsplatz, Wunsch der Angestellten und der Finanzmitteln ab. Entscheidend sind auch die Politik der Organisation im Bereich der (Fremdsprachen)Ausbildung und die Anordnung des Vorgesetzten. Beinahe ein Drittel der Organisationen stellt den Fremdsprachenbedarf der Angestellten gar nicht fest.

Die Ergebnisanalyse widerlegte die Hypothese, dass die Organisationen der öffentlichen Verwaltung Fremdsprachenkurse der Angestellten nicht finanzieren. Der größte Teil der Organisationen bezahlt ihnen die Fremdsprachenkurse ganz.

Die Angestellten in der öffentlichen Verwaltung haben den größten Bedarf an allgemeinen Fremdsprachen- und Konversationskursen. Spezialisierte Kurse benötigen sie zur Aneignung an die Amtssprache und Fachterminologie aus dem Bereich der Stevern, der EU-Vorschriften und Ausschreibungen, der Rechtssprache, Fachbegriffe aus dem Bereich der Arbeit, Beschäftigung, Sicherheit und Gesundheit bei der Arbeit, Verhandlungen und Vorbereitungen auf die Ausschreibungen und Arbeit mit den ausländischen Kunden (bei der Erledigung der standesamtlichen Angelegenheiten, Ausstellung der Führerscheine und Zulassungen). Fast die Hälfte der Organisationen (43\%) benötigen spezialisierte Englischkurse mehr als spezialisierte Deutschkurse (24\%).

Angestellte, denen Fremdsprachenausbildung ermöglicht worden ist, besuchen einen Fremdsprachenkurs mehrere Monate jährlich, da dir nur einige Stunden wöchentlich stattfinden. Intensivkurse werden in längeren Intervallen mit mehreren Unterrichtsstunden täglich durchgeführt. 
Ein- oder zweitägige Fremdsprachen-Workshops finden fast nie statt. In den meisten Organisationen verlaufen Fremdsprachenkurse während der Arbeitszeit.

Die meisten Befragten antworteten, dass sich der Bedarf an Fremdsprachen und Fremdsprachenausbildung im öffentlichen Sektor erhöht. 27\% der Organisationen waren der Meinung, dass sich der Bedarf verändert, während 3,2\% der Organisationen davon überzeugt waren, dass er zurückgeht.

\section{Schlussfolgerung}

Die slowenische öffentliche Verwaltung war die ganze Zeit (besonders in den letzten Jahren) verschiedenen Formen der Fremdsprachenbedarfe ausgesetzt. Dafür sind die meisten Gründe auf die historischen (ehemaliger Staat), politischen (Unabhängigkeit) und strukturellen Veränderungen (EU) zurückzuführen. Die Tätigkeiten der Angestellten im öffentlichen Dienst sind besonders von der Einbettung ins europäische Geschehen und vielfach der "Lebensabhängigkeit" von den europäischen Direktiven und Ausschreibungen geprägt. Trotzdem ist das Bewusstsein über den Bedarf an fremdsprachlichen Geschäftstätigkeiten in der öffentlichen Verwaltung relativ niedrig. Voraussichtlich hängt das damit zusammen, dass sich die Angestellten in der öffentlichen Verwaltung über die Bedeutung und Vorteile der Fremdsprachenkenntnisse bei der Wahrnehmung von Arbeitsaufgaben nicht bewusst sind.

Dies bestätigt die Feststellung, dass bei uns in der Öffentlichkeit noch immer die Überzeugung herrscht, dass Fremdsprachenkenntnisse von zentraler Bedeutung für die hochwertige Wahrnehmung von Arbeitsaufgaben der Angestellten im öffentlichen Dienst sind und dies in den meisten Fällen mit der einheimischen Bevölkerung zusammenhängt. Jedoch die Untersuchung zeigte ein anderes Bild.

In der Wirtschaft ist das Bewusstsein über die Bedeutung der Fremdsprachen für die erfolgreiche Kommunikation und Zusammenarbeit in verschiedenen Bereichen hoch. Immer mehr Unternehmen bemühen sich darum, die Fremdsprachenkenntnisse ihrer Angestellten zu verbessern, weil sie eng mit der Fähigkeit der Internationalisierung zusammenhängen, und einer der wichtigsten Werkzeuge zur Erlangung von wichtigen geschäftlichen und wissenschaftlichen Informationen sowie zusätzlichen finanziellen Mitteln (z.B. aus den europäischen Fonds) sind. 
Margit Horvath, Manica Danko, Polonca Kovač, Janez Stare

Fremdsprachenausbildung - Bedarfs- und Erfahrungsanalyse der Fremdsprachenausbildung in der slowenischen öffentlichen Verwaltung

Auch die Europäische Kommission hebt die Bedeutung der Mehrsprachigkeit für den Geschäftserfolg auf dem Globalmarkt hervor. Neben dem Englischen, das als Weltsprache die führende Rolle behalten hat, gibt es auch andere Sprachen, die für die Unterscheidung zwischen dem Durchschnitt und der Excellence ausschlaggebend sind und den Wettbewerbsvorteil bringen.

In diesem Sinne müsste auch der Staat als wirtschaftlicher Arbeitgeber handeln und der Mehrsprachigkeit eine größere Bedeutung zuschreiben. Aufgrund unserer Anwesenheit in der EU, stellen die Fremdsprachenkenntnisse nicht nur die Brücke zu anderen europäischen Kulturen dar, sondern schaffen für die Organisationen in der öffentlichen Verwaltung auch viele Möglichkeiten für neue Geschäftsgelegenheiten. Die Angestellten im öffentlichen Dienst benötigen die Fremdsprachenkenntnisse nicht nur, um mit den ausländischen Kunden erfolgreich Kontakte aufzunehmen, sondern auch als ein wichtiges Werkzeug für die Verbindung mit den auswärtigen "Märkten" und profitable Geschäfte mithilfe von europäischen Projekten.

Margit Horvath bildete sich neben dem Studium der Anglistik und Germanistik an der Philosophischen Fakultät der Universität Ljubliana auch an der Universität Heidelberg und Graz im DaF-Bereich weiter. Im Jahr 2002 schloss sie den postgradualen Universitätslehrgang Executive MBA an der Donau-Universität Krems ab. Ihre Forschungsarbeit konzentriert sich auf die Vergleichsanalyse von deutsch-slowenischen Texten im Bereich der Wirtschafts- und Verwaltungswissenschaften und die Qualitätssteigerung des Fremdsprachenunterrichts. Sie ist an der Fakultät für Verwaltungswissenschaften als Dozentin für Deutsch tätig.

Manica Danko ist Lektorin für Englisch als Fachsprache an der Fakultät für Verwaltungswissenschaften. Sie schloss das Magisterstudium TESOL an der Universität Manchester im Bereich der Erstellung von universitären Fremdsprachenlehrwerken ab. Ihre Forschungstätigkeit bezieht sich auf die Qualität des Fremdsprachenunterrichts und die Vernetzung der Studienfächer im Hochschulwesen. Sie ist auch als Lehrwerkautorin tätig. 
Margit Horvath, Manica Danko, Polonca Kovač, Janez Stare

Fremdsprachenausbildung - Bedarfs- und Erfahrungsanalyse der

Fremdsprachenausbildung in der slowenischen öffentlichen Verwaltung

Dr. Polonca Kovač ist Forscherin und Universitätsdozentin für Rechtsordnung und Reformen der öffentlichen Verwaltung und Verwaltungsprozessrecht. Ihr Doktorstudium absolvierte sie an der Juristischen Fakultät der Universität Ljubliana. Sie ist Autorin, Koautorin und Redakteurin zahlreicher Monografien (z.B. Beurteilung der Regulationseffekte 2009; Verwaltungsverfahren und Verwaltungsstreitigkeit, 2010, Entwicklung der slowenischen öffentlichen Verwaltung 1991-2011) und Beiträge in Periodika und auf Wissenschafts- und Fachkonferenzen im In- und Ausland.

Dr. Janez Stare ist Universitätsdozent für Organisation des öffentlichen Sektors an der Fakultät für Verwaltungswissenschaften der Universität Ljubliana. Er ist Studienfachleiter auf Bachelor- und Master-Ebene und nimmt auch Aufgaben als Prodekan für Studienangelegenheiten wahr. Zudem übt er auch die Funktion als Lehrstuhlinhaber für Organisation und Informatik aus. Der Fokus seiner wissenschaftlichen, pädagogischen und Beratungstätigkeit liegt im Bereich der Führung, Personalverwaltung und Organisation. Sein Expertenwissen eignete er sich im Inland und an renommierten Institutionen im Ausland an. Er wirkt als Projektleiter oder -mitarbeiter mit und beteiligt sich an verschiedenen Wissenschafts- und Forschungstreffen. Seit 2017 ist er Vorsitzender des Beamtenrates. 
Margit Horvath, Manica Danko, Polonca Kovač, Janez Stare

Fremdsprachenausbildung - Bedarfs- und Erfahrungsanalyse der Fremdsprachenausbildung in der slowenischen öffentlichen Verwaltung

\section{Literatur und Quellennachweis}

- Bundesministerium für Unterricht, Kunst und Kultur (BMUKK), Österreich, Schlick, Klaus, Diskussionsforum 4, (2010). Mobilität und Fremdsprachen in einem europäischen Bildungsraum ohne Grenzen, 1-15. Erworben am 30. 1. 2012, http://www.bmukk.gv.at/medienpool/8169/Forum4.pdf.

- Čebron, N. (1998). Status quo of ESP at the tertiary education level in Slovenia - a focus on the University of Ljubliana. V: Čebron, N. (ur.). New challenges in teaching ESP in Slovenia / IATEFL Slovenia, lst National ESP Conference (23-31), Portorož, 11-13 June 1998.

- Danko, M., Stare, J. \& Horvath, M. (2009). Analiza in ugotavljanje potreb študentov in diplomantov Fakultete za upravo (FU/NUŠ) na področju jezikovnega izobraževanja oz. pri predmetih angleški in nemški jezik.

- Djurić, M. (1999). Kako lahko preoblikujemo tradicijo ESP/EAP v Sloveniji. Vestnik 33(1-2), 7-22.

- Djurić, M. et al. (2008). Tuji jeziki stroke v slovenskem visokem šolstvu: poimenovanje, ravni, obseg, cilji in učna gradiva. V: Skela, J. (ur.). Učenje in poučevanje tujih jezikov na Slovenskem (523-525). Ljubljana: Tangram.

- EC.EUROPA (2009). Languages of Europe. Erworben am 30. 8. 2009, http://ec.europa.eu/education/languages/languages-of-europe/ doc135_en.htm.

- Education and Training 2020 (ET 2020). The Council of Europe, (2009). Erworben am 30. 1. 2012, http://europa.eu/legislation_summaries/ education_training_youth/general_framework/cl1086_en.htm.

- Erbič D. (2008): Jezikovna ureditev Evropske unije in posledice članstva $v$ EU za slovenski jezik. V: Ivšek, M. (ur.) Jeziki v izobraževanju. Zbornik prispevkov konference (107-1 10), Ljubljana, 25.-26. septembra 2008. Ljubljana: Zavod RS za šolstvo.

- Goethe Institut (Gl) Ljubljana, (2009). Razlogi za učenje nemščine, pogovor s Hansom-Jürgenom Krummom. Erworben am 24. 1. 2012, http://www.goethe.de/ins/si/liu/lhr/liu_lhr_gruende/s/4568512.htm.

- Goethe-Institut (GI). Der gemeinsame Europäische Referenzrahmen in seinem politischen und bildungspolitischen Kontext, Was bedeutete Mehrsprachigkeit? Erworben am 24. 1. 2012, http://www.goethe.de/z/50/commeuro/.

- Grmek, A. \& Rejec, S. (2005). Nemščina v slovenskem visokošolskem prostoru. Erworben am 3. 8. 20009, http://www2.arnes.si/ sdunjl/ SCHAUREIN_18_povzetki.pdf.

- Horvath, M. (2008). Studienbegleitender Deutschunterricht in Slowenien. V: Lévy-Hillerich, D. \& Serena, S. (ur.). Studienbegleitender Deutschunterricht in Europa. Rückblick und Ausblick: Versuch einer Standortbestimmung (372414), Roma: Lingue d’Europa e del Mediterraneo, Aracne Editrice (izdano 2009). 
Margit Horvath, Manica Danko, Polonca Kovač, Janez Stare

Fremdsprachenausbildung - Bedarfs- und Erfahrungsanalyse der

Fremdsprachenausbildung in der slowenischen öffentlichen Verwaltung

- Jakoš, Z. (2007). The role of ESP in the newly developed Bologna study programmes at Slovene faculties. In Graf, E.-M. \& James Allen (ur.). English studies in flux (149-157). Tübingen: Gunter Narr Verlag.

- Jerovšek. T. \& Kovač, P. (2010). Upravni postopek in upravni spor. Ljubljana: Fakulteta za upravo.

- Jurković, V. et al. (2008). Status učitelja tujega jezika stroke v visokem šolstvu. V: Inter alia, zbornik prispevkov s posveta SDUTSJ (23. 11 .2007).

- Jurković, V. \& Djurić, M. (2008): Tuji jeziki stroke v slovenskem visokem šolstvu. V: Ivšek, M. (ur.). Jeziki v izobraževanju. Zbornik prispevkov konference (215-222), Ljubljana, 25.-26. September 2008. Ljubljana: Zavod Republike Slovenije za šolstvo.

- Komac, M. (2007). Priseljenci, Študije o priseljevanju in vključevanju v slovensko družbo. Inštitut za narodnostna vprašanja.

- Kovač, P., Rakar, I. \& Remic, M. (2012): Upravno-procesne dileme o rabi ZUP, 2. del - vprašanja iz prakse z odgovori, sodno prakso in uvodno študijo (v tisku). Ljubljana: Uradni list RS.

- Ministrstvo Republike Slovenije za šolstvo in šport (MSS), Skupni evropski jezikovni okvir: učenje, poučevanje, ocenjevanje, (2011). Erworben am 24. 1. 2012, http://www.mss.gov.si/fileadmin/mss.gov.si/pageuploads/ podrocje/razvoi_solstva/Jeziki/Publikacija_SEJO_komplet.pdf.

- Nancy Declaration. Multilingual Universities for a Multilingual Europe Open to the World, (2006). Erworben am 30. 1. 2012,

http://www.campuseuropae.org/en/support/docs/langlearning/enlu_nancy_ declaration.pdf.

- Pregled posledic odsotnosti skladne tujejezikovne politike na vseh ravneh izobraževanja $\vee$ RS ter predlogi za izboljšavo ugotovljenega stanja. Erworben am 16.1.2012, http://www.sdutsj.edus.si/tujejezikovna_politika.html.

- Statistični urad Republike Slovenije (2011). Selitveno gibanje, 2009, končni podatki. Erworben am 24. 1. 2012, http://www.stat.si/novica_prikazi.aspx?id=3362.

- Stare, J. (2007). Rezultat dela na projektu v okviru ciljno raziskovalnega programa (CRP) »Konkurenčnost Slovenije2006-2013«, Naslov projekta: Določitev kompetenc zaposlenih v javni upravi. Erworben am 24. 1. 2012, Hp://www.miu.gov.si/fileadmin/mju.gov.si/pageuploads/mju_dokumenti/DO K/dolocitev_kompetenc_zaposlenih_v_javni_upravi.pdf.

- Šturm, L. (ur.) et al. (2011). Komentar Ustave Republike Slovenije - dopolnitev A. Brdo: Fakulteta za državne in evropske študije.

- Svetličič, M. (2011a). Kompetence za ekonomsko diplomacijo in mednarodno poslovanje; konvergenca ali divergenca? Uprava 9(1), 123-141.

- Svetličič, M. (2011b). Diplomacija in mednarodno poslovanje v 21 . stoletju: komplementarnost ali nadomestnost. Teorija in praksa 48(3), 604-627.

- Svetlin-Gvardjančič, P. et. al (2009). Strani jezik struke u bolonjskom procesu: status predmeta in organizacija nastave. (str. 721-735). V: Vučo, J., 
Margit Horvath, Manica Danko, Polonca Kovač, Janez Stare

Fremdsprachenausbildung - Bedarfs- und Erfahrungsanalyse der Fremdsprachenausbildung in der slowenischen öffentlichen Verwaltung

Ignjačević, A. \& Mirić, M.(ur.). Jezik struke: Teorija i praksa. Zbornik radova. Univerzitet u Beogradu.

- Ustava RS, Ur. I. RS, št. 33/91-1, 42/97, 66/04.

- Verlič Christensen, B. (2000). Migracijska politika Evropske skupnosti in Slovenija. Teorija in praksa 37(6), 1117-1131. Erworben am 30. 8. 2009, http://dk.fdv.uni-li.si/tip/tip20006verlicchristensen.PDF.

- Zakon o javni rabi slovenščine Ur. I. RS, št. 86/04.

- Zakon o splošnem upravnem postopku (ZUP), Ur. I. RS, št. 80/99 in nasl.

- Zavašnik, M. (2006). Analiza potreb učiteljev angleškega strokovnega jezika z vidika niihovih specifičnih sposobnosti v okviru usposabljanja in izpopolnjevanja. Neobjavljen doktorat. Ljubljana: Filozofska fakulteta. 